\title{
Exploring the Higgs sector of a most natural NMSSM and its prediction on Higgs pair production at the LHC
}

\author{
Junjie Cao, ${ }^{a, b}$ Dongwei Li, ${ }^{a, c}$ Liangliang Shang, ${ }^{a, d}$ Peiwen $\mathbf{W u}{ }^{d}$ and Yang Zhang ${ }^{d}$ \\ ${ }^{a}$ Department of Physics, Henan Normal University, \\ Xinxiang 453007, China \\ ${ }^{b}$ Center for High Energy Physics, Peking University, \\ Beijing 100871, China \\ ${ }^{c}$ Department of Foundation, Henan Police College, \\ Zhengzhou 450000, China \\ ${ }^{d}$ State Key Laboratory of Theoretical Physics, Institute of Theoretical Physics, \\ Academia Sinica, Beijing 100190, China \\ E-mail: junjiec@itp.ac.cn, 353232505@qq.com, shlwell1988@gmail.com, \\ pwwu@itp.ac.cn, zhangyang@itp.ac.cn
}

AbStRACT: As a most natural realization of the Next-to Minimal Supersymmetry Standard Model (NMSSM), $\lambda$-SUSY is parameterized by a large $\lambda$ around one and a low $\tan \beta$ below 10. In this work, we first scan the parameter space of $\lambda$-SUSY by considering various experimental constraints, including the limitation from the Higgs data updated by the ATLAS and CMS collaborations in the summer of 2014, then we study the properties of the Higgs bosons. We get two characteristic features of $\lambda$-SUSY in experimentally allowed parameter space. One is the triple self coupling of the SM-like Higgs boson may get enhanced by a factor over 10 in comparison with its SM prediction. The other is the pair production of the SM-like Higgs boson at the LHC may be two orders larger than its SM prediction. All these features seems to be unachievable in the Minimal Supersymmetric Standard Model and in the NMSSM with a low $\lambda$. Moreover, we also find that naturalness plays an important role in selecting the parameter space of $\lambda$-SUSY, and that the Higgs $\chi^{2}$ obtained with the latest data is usually significantly smaller than before due to the more consistency of the two collaboration measurements.

KeYWords: Supersymmetry Phenomenology

ARXIV EPRINT: 1409.8431 


\section{Contents}

1 Introduction $\quad 1$

2 Higgs sector in $\lambda$-SUSY and our scan strategy 3

2.1 Higgs sector in NMSSM with a large $\lambda \quad 3$

2.2 Strategy in scanning the parameter space of $\lambda$-SUSY 6

3 Properties of $h_{1}$ and $h_{2} \quad 10$

$\begin{array}{ll}3.1 \text { Properties of the lightest CP-even Higgs boson } & 11\end{array}$

3.2 Properties of the next-to-lightest CP-even Higgs boson 13

$\begin{array}{llr}4 & \text { Higgs pair production at the LHC } & 18\end{array}$

5 Summary and conclusions $\quad 22$

\section{Introduction}

Compared with the situation in 2012, the existence of a new scalar with mass around $125 \mathrm{GeV}$ has been further corroborated by the ATLAS and CMS collaborations with a local statistical significance reaching $9 \sigma$ and more than $7 \sigma$ respectively [1-4]. Especially, recently both the collaborations updated their measurements on the properties of the scalar by using the detector calibration in the event reconstruction [5-8], and as indicated by their published data, the two group measurements now agree with each other in a much better way. So far the mass of the scalar is rather precisely determined, and its other properties, albeit still with large experimental uncertainties, coincide with those of the Higgs boson predicted by the Standard Model (SM). Nevertheless, the issue of whether this particle is the SM Higgs boson is still open, and indeed there are some motivations, such as the gauge hierarchy problem and the intriguing slight excess of the di-photon signal for the scalar over the SM prediction, which now is $\mu_{\gamma \gamma}=1.17 \pm 0.27$ by the ATLAS measurement [5] and $\mu_{\gamma \gamma}=1.13 \pm 0.24$ by the CMS measurement [8], to consider new physics interpretation of this particle. Studies in this direction have been performed intensively in supersymmetric theories (SUSY) (for MSSM explanation of the $125 \mathrm{GeV}$ scalar, see for example [9-20]; for NMSSM explanation of the $125 \mathrm{GeV}$ scalar, see for example [21-34]), which are considered as the most promising new physics candidates due to their capability to unify the gauge couplings, provide a viable Dark Matter candidate as well as stabilize the weak scale in a much better way than the SM. These studies indicated that, although in the Minimal Supersymmetric Standard Model (MSSM) there exists a broad parameter space to fit the Higgs data quite well $[35,36]$, the mass of the observed particle leads to a well-known tension with naturalness since it is much larger than the upper bound of the tree-level 
Higgs mass, which is controlled by the weak gauge coupling due to the structure of the model [37]. This tension led to a revival of interest in non-minimal realizations of SUSY at the weak scale. Arguably the simplest among such extended constructions is the Nextto-Minimal Supersymmetric Standard Model (NMSSM) [38-40], which will be the focus of this paper.

In the NMSSM, the particle content is extended by including a gauge singlet superfield $\hat{S}$ with its interaction with the MSSM Higgs superfields $\hat{H}_{u}$ and $\hat{H}_{d}$ taking the form $\lambda \hat{S} \hat{H}_{u} \cdot \hat{H}_{d}\left(\hat{H}_{u} \cdot \hat{H}_{d} \equiv \epsilon_{a b} \hat{H}_{u}^{a} \hat{H}_{d}^{b}\right.$ is $\mathrm{SU}(2)$ index contraction) [38-40]. The inclusion of the singlet allows the quartic terms of the Higgs potential to get a new contribution, which is proportional to $\lambda^{2}$. This will lift the tree-level Higgs mass and consequently alleviate the tension [37]. In fact, it is due to this advantage that the NMSSM was widely adopted to interpret the LHC results [21-34]. While on the other hand, since $\lambda$ is up bounded by the perturbativity of the theory below the grand unification scale, i.e. $\lambda \lesssim 0.7$, the size of the lift is mild and so the naturalness problem is only partially addressed. Under such a situation, $\lambda$-SUSY which corresponds to the NMSSM with a relatively large $\lambda$ around 1 was recently emphasized [41-48]. As suggested by the pioneer works in this direction, the NMSSM may still maintain the grand unification and perturbativity for such a $\lambda$ if an appropriate new dynamics is implemented at a certain ultraviolet energy scale [49-53]. Moreover, it was pointed out that in $\lambda$-SUSY, the sensitivity of the weak scale to the scalar top quark (stop) mass is reduced by a factor of $\sim(g / \lambda)^{2}$ in comparison with the MSSM ( $g$ is the SM weak gauge coupling), which means that the lower bound on the stop mass imposed by the LHC direct searches has a weaker implication on fine-tuning in this model than in the MSSM or the NMSSM with a low $\lambda$ [53]. In this sense, $\lambda$-SUSY has been treated as a simplest and meanwhile most natural realization of SUSY at weak scale. ${ }^{1}$

In $\lambda$-SUSY, the phenomenology in Higgs sector is rather special. Firstly, since the tree-level mass of the SM-like Higgs boson (denoted by $h$ hereafter) may be easily higher than $125 \mathrm{GeV}$, the boson must have sizable singlet and/or non-SM doublet components. Consequently, its couplings might deviate significantly from their SM predictions, which will be constrained by the recently updated Higgs data [46-48]. Secondly, unlike the MSSM where a large $\tan \beta$ is preferred to enhance the tree-level Higgs mass, $\tan \beta$ in $\lambda$-SUSY must be rather low, i.e. $\tan \beta \lesssim 4$, to coincide with the electro-weak precision data $[53,54]$. In this case, the constraints of the LHC direct search for neutral non-SM Higgs bosons by $\tau \bar{\tau}$ channel are weakened [56, 57], and the non-SM Higgs bosons may be significantly lighter than those of the MSSM. This will result in a rather different phenomenology [55], but so far is paid little attention in literature. Thirdly, as we mentioned before, the quartic terms of the Higgs potential are altered greatly in $\lambda$-SUSY so that the interactions among the physical Higgs particles may be significantly strengthened [46, 47]. Under such a situation, the $h h$ production may be greatly enhanced by the mediation of the non-SM Higgs bosons, which may decay into the Higgs pair dominantly [58-61], and/or by the trilinear self coupling of the SM-like Higgs boson, which may be much stronger than the

\footnotetext{
${ }^{1}$ We would like to mention that $\lambda$-SUSY is not the only setup to improve the fine-tuning in the singlet extensions of the MSSM. In fact, in some more complex frameworks such as the GNMSSM [137] and the DiracNMSSM [138], the fine-tuning problem can also be greatly alleviated in a nice way [137, 139-141].
} 
SM prediction in some parameter region of the $\lambda$-SUSY. Considering the importance of the pair production in extracting the Higgs self coupling information, such enhancement effects should be investigated carefully. Noting above features, we in this work first consider various experimental constraints on $\lambda$-SUSY, then we explore the Higgs sector by focusing on the properties of the lightest and the next-to-lightest CP-even Higgs bosons. We also investigate how large the Higgs pair production rate may get enhanced in $\lambda$-SUSY.

This work is organized as follows. In section 2 , we recapitulate the framework of $\lambda$ SUSY and the features of its Higgs sector. Then we scan its parameter space by considering various constraints to get physical parameter points. In section 3, we investigate the predictions of these points on the properties of the lightest and the next-to-lightest CPeven Higgs boson, such as their couplings and decay rates, to show their distinctive features. In section 4, we study the SM-like Higgs pair production process, and point out that its rate in $\lambda$-SUSY may be enhanced by a factor of 100 over its SM prediction, which is hardly achieved in the MSSM. Finally, we draw our conclusions.

\section{Higgs sector in $\lambda$-SUSY and our scan strategy}

\subsection{Higgs sector in NMSSM with a large $\lambda$}

The NMSSM extends the MSSM with one gauge singlet superfield $\hat{S}$, and since it aims at solving the $\mu$ problem of the MSSM, a $Z_{3}$ discrete symmetry under which the Higgs superfields $\hat{H}_{u}, \hat{H}_{d}$ and $\hat{S}$ are charged is implemented in the construction of the superpotential to avoid the appearance of parameters with mass dimension. As a result, its superpotential is given by [38-40]

$$
W^{\mathrm{NMSSM}}=W_{F}+\lambda \hat{H}_{u} \cdot \hat{H}_{d} \hat{S}+\frac{1}{3} \kappa \hat{S^{3}},
$$

where $W_{F}$ is the superpotential of the MSSM without the $\mu$-term, and $\lambda, \kappa$ are all dimensionless parameters describing the interactions among the superfields. The scalar potential for the Higgs fields $H_{u}, H_{d}$ and $S$ is given by the sum of the usual F- and D-term contributions, and the soft breaking terms:

$$
V_{\mathrm{soft}}^{\mathrm{NMSSM}}=\tilde{m}_{u}^{2}\left|H_{u}\right|^{2}+\tilde{m}_{d}^{2}\left|H_{d}\right|^{2}+\tilde{m}_{S}^{2}|S|^{2}+\left(\lambda A_{\lambda} S H_{u} \cdot H_{d}+\frac{1}{3} \kappa A_{\kappa} S^{3}+\text { h.c. }\right) .
$$

In all, the Higgs sector Lagrangian contains 7 free parameters, which include

$$
p_{i}^{\text {susy }}=\left\{\lambda, \kappa, \tilde{m}_{u}^{2}, \tilde{m}_{d}^{2}, \tilde{m}_{S}^{2}, A_{\lambda}, A_{\kappa}\right\} .
$$

With the scalar potential expressed in term of the fields $H_{u}, H_{d}$ and $S$, it is not easy to see its particle implication on the LHC results. To improve such a situation, one usually introduces following combinations of the Higgs fields [38-40]

$$
H_{1}=\cos \beta H_{u}+\varepsilon \sin \beta H_{d}^{*}, \quad H_{2}=\sin \beta H_{u}-\varepsilon \cos \beta H_{d}^{*}, \quad H_{3}=S,
$$


where $\varepsilon_{12}=-\varepsilon_{21}=1, \varepsilon_{11}=\varepsilon_{22}=0$ and $\tan \beta \equiv v_{u} / v_{d}$ with $v_{u}$ and $v_{d}$ representing the vacuum expectation values of the fields $H_{u}$ and $H_{d}$. In this representation, $H_{i}(i=1,2,3)$ are given by

$$
H_{1}=\left(\begin{array}{c}
H^{+} \\
\frac{S_{1}+i P_{1}}{\sqrt{2}}
\end{array}\right), \quad H_{2}=\left(\begin{array}{c}
G^{+} \\
v+\frac{S_{2}+i G^{0}}{\sqrt{2}}
\end{array}\right), \quad H_{3}=v_{s}+\frac{1}{\sqrt{2}}\left(S_{3}+i P_{2}\right) .
$$

These expressions indicate that the field $\mathrm{H}_{2}$ corresponds to the SM Higgs field with $\mathrm{G}^{+}$ and $G^{0}$ denoting Goldstone bosons, and $S_{2}$ representing the SM Higgs field (so it should make up the dominant component of the observed scalar as suggested by the LHC data), and the field $H_{1}$ represents a new $\mathrm{SU}(2)_{L}$ doublet scalar field, which has no tree-level couplings to the $\mathrm{W} / \mathrm{Z}$ bosons. Eq. (2.5) also indicates that the Higgs sector of the NMSSM includes three CP-even mass eigenstates, which are the mixtures of the fields $S_{1}, S_{2}$ and $S_{3}$, two CP-odd mass eigenstates composed by the fields $P_{1}$ and $P_{2}$, as well as one charged Higgs $H^{+}$.

In practical application, it is usually more convenient to use [38-40]

$$
\lambda, \quad \kappa, \quad \tan \beta, \quad \mu, \quad M_{A}, \quad M_{P},
$$

as input parameters, where $\tilde{m}_{u}^{2}, \tilde{m}_{d}^{2}$ and $\tilde{m}_{S}^{2}$ in eq. (2.3) are traded for $m_{Z}, \tan \beta \equiv v_{u} / v_{d}$ and $\mu \equiv \lambda v_{s}$ by the potential minimization conditions, and $A_{\lambda}$ and $A_{\kappa}$ are replaced by the squared masses of the CP-odd fields $P_{1}$ and $P_{2}$, which are given by

$$
M_{A}^{2}=\frac{2 \mu}{\sin 2 \beta}\left(A_{\lambda}+\kappa v_{s}\right), \quad M_{P}^{2}=\lambda^{2} v^{2}\left(\frac{M_{A}}{2 \mu / \sin 2 \beta}\right)^{2}+\frac{3}{2} \lambda \kappa v^{2} \sin 2 \beta-3 \kappa v_{s} A_{\kappa} .
$$

Note that $M_{A}$ and $M_{P}$ represent the tree-level CP-odd particle masses only when the mixing between $P_{1}$ and $P_{2}$ vanishes.

With this set of input parameters, the mass matrix for CP-even Higgs bosons in the basis $\left(S_{1}, S_{2}, S_{3}\right)$ is given by [38-40]

$$
\begin{aligned}
& \mathcal{M}_{S, 11}^{2}=M_{A}^{2}+\left(m_{Z}^{2}-\lambda^{2} v^{2}\right) \sin ^{2} 2 \beta, \\
& \mathcal{M}_{S, 12}^{2}=-\frac{1}{2}\left(m_{Z}^{2}-\lambda^{2} v^{2}\right) \sin 4 \beta, \\
& \mathcal{M}_{S, 13}^{2}=-\left(\frac{M_{A}^{2}}{2 \mu / \sin 2 \beta}+\kappa v_{s}\right) \lambda v \cos 2 \beta, \\
& \mathcal{M}_{S, 22}^{2}=m_{Z}^{2} \cos ^{2} 2 \beta+\lambda^{2} v^{2} \sin ^{2} 2 \beta, \\
& \mathcal{M}_{S, 23}^{2}=2 \lambda \mu v\left[1-\left(\frac{M_{A}}{2 \mu / \sin 2 \beta}\right)^{2}-\frac{\kappa}{2 \lambda} \sin 2 \beta\right], \\
& \mathcal{M}_{S, 33}^{2}=\frac{1}{6} \lambda^{2} v^{2}\left(\frac{M_{A}}{\mu / \sin 2 \beta}\right)^{2}+4\left(\kappa v_{s}\right)^{2}-\frac{1}{3} M_{P}^{2},
\end{aligned}
$$

and the corresponding mass eigenstates $h_{i}(i=1,2,3)$ are obtained by diagonalizing the mass matrix:

$$
h_{i}=\sum_{j=1}^{3} V_{i j} S_{j}
$$


where $V_{i j}$ denotes the rotation matrix. In the following, we assume $m_{h_{3}}>m_{h_{2}}>m_{h_{1}}$, and call the state $h_{i}$ the SM-like Higgs boson (non-SM doublet Higgs boson) if $\left|V_{i 2}\right|^{2}>0.5$ $\left(\left|V_{i 1}\right|^{2}>0.5\right)$. Moreover, in order to present our results in a compact way we define $\bar{S}_{i}=V_{i 3}$ and $\bar{D}_{i}=V_{i 1}$ with $\left|\bar{S}_{i}\right|^{2}$ and $\left|\bar{D}_{i}\right|^{2}$ representing the singlet and non-SM doublet components in the physical state $h_{i}$ respectively. With this notation, the couplings of $h_{i}$ with vector bosons and fermions are given by

$$
\begin{gathered}
C_{h_{i} V V} / S M=\operatorname{Sign}\left(V_{i 2}\right) \sqrt{1-\bar{D}_{i}^{2}-\bar{S}_{i}^{2}}, \quad V=Z, W, \\
C_{h_{i} \bar{u} u} / S M=\bar{D}_{i} \cot \beta+\operatorname{Sign}\left(V_{i 2}\right) \sqrt{1-\bar{D}_{i}^{2}-\bar{S}_{i}^{2}}, \\
C_{h_{i} \bar{d} d} / S M=-\bar{D}_{i} \tan \beta+\operatorname{Sign}\left(V_{i 2}\right) \sqrt{1-\bar{D}_{i}^{2}-\bar{S}_{i}^{2}},
\end{gathered}
$$

where the denominator $S M$ means the corresponding Higgs coupling in the SM. We also have following sum rules

$$
\begin{aligned}
\bar{D}_{1}^{2}+\bar{D}_{2}^{2}+\bar{D}_{3}^{2} & =1, \\
\bar{S}_{1}^{2}+\bar{S}_{2}^{2}+\bar{S}_{3}^{2} & =1 .
\end{aligned}
$$

The expression of $\mathcal{M}_{S, 22}^{2}$ in eq. (2.8) indicates that, without the mixings of the CP-even states, the SM-like Higgs mass at tree level is given by

$$
m_{h, \text { tree }}^{2} \simeq m_{Z}^{2} \cos ^{2} 2 \beta+\lambda^{2} v^{2} \sin ^{2} 2 \beta,
$$

where the last term on the right side is peculiar to any singlet extension of the MSSM [3840], and its effect is to enhance the mass. Obviously, if the NMSSM is a natural theory, $m_{h \text {,tree }}$ should lie near $125 \mathrm{GeV}$, but in practice, this is not so since the perturbativity of the theory up to GUT scale has required $\lambda \lesssim 0.7$ so that $m_{h \text {,tree }}^{2}$ usually falls far short of the desired value. For example, given $\tan \beta=3$ and $\lambda=0.7$, one can get $m_{h \text {,tree }} \simeq 97 \mathrm{GeV}$, which means $\Delta^{2} / m_{h \text {,tree }}^{2} \simeq 2 / 3$ for the top-stop loop correction $\Delta^{2}$ in order to predict the $125 \mathrm{GeV}$ Higgs boson in no mixing case. Confronted with such a situation, $\lambda$-SUSY which corresponds to the NMSSM with a large $\lambda$ around one was proposed [49-53]. This theory is based on the hypothesis that the NMSSM with a large $\lambda$ is only an effective Lagrangian at the weak scale, and an appropriate structure of superfields intervenes at an ultraviolet energy scale (usually chosen at $10 \mathrm{TeV}$ ) so that the virtues of SUSY such as the grand unification of the gauge couplings are maintained. Under this assumption, the values of $\lambda$ and $\kappa$ at weak scale are relaxed by [48]

$$
0.17 \lambda^{2}+0.26 \kappa^{2} \lesssim 1
$$

In $\lambda$-SUSY, two fine tuning quantities are defined to measure the naturalness of the theory [48]:

$$
\Delta_{Z}=\max _{i}\left|\frac{\partial \log m_{Z}^{2}}{\partial \log p_{i}}\right|, \quad \Delta_{h}=\max _{i}\left|\frac{\partial \log m_{h}^{2}}{\partial \log p_{i}}\right|,
$$

where $p_{i}$ denotes SUSY parameters at the weak scale, and it includes the parameters listed in eq. (2.3) and top quark Yukawa coupling $Y_{t}$ with the latter used to estimate the 
sensitivity to stop mass. Obviously, $\Delta_{Z}\left(\Delta_{h}\right)$ measures the sensitive of the weak scale (the Higgs mass) to SUSY parameters, and the larger its value becomes, the more tuning is needed to get the corresponding mass. In our calculation, we calculate $\Delta_{Z}$ and $\Delta_{h}$ by the formulae presented in [62] and [48] respectively.

Throughout this work, we consider the lightest CP-even Higgs boson as the SM-like Higgs boson. The possibility that the next-to-lightest CP-even Higgs boson corresponds to the SM-like Higgs boson is theoretically less appealing since $m_{h \text {,tree }}$ in $\lambda$-SUSY usually exceeds $125 \mathrm{GeV}$, and the mixing between $S_{2}$ and $S_{3}$ can further push up the mass so that the theory has more tuning to get the Higgs boson mass. Our numerical scan checked this point.

\subsection{Strategy in scanning the parameter space of $\lambda$-SUSY}

In this work, we first perform a comprehensive scan over the parameter space of $\lambda$-SUSY by considering various experimental constraints. Then for the surviving samples we investigate the features of its Higgs sector. In order to simplify our analysis, we make following assumptions about some unimportant SUSY parameters:

- First, we fix all soft breaking parameters for the first two generation squarks at $2 \mathrm{TeV}$. For the third generation squarks, considering that they can affect significantly the mass of the SM-like Higgs boson, we set free all soft parameters in this sector except that we assume $m_{U_{3}}=m_{D_{3}}$ for right-handed soft breaking masses and $A_{t}=A_{b}$ for soft breaking trilinear coefficients.

- Second, since we require $\lambda$-SUSY to explain the discrepancy of the measured value of the muon anomalous magnetic moment from its SM prediction, we assume all soft breaking parameters in the slepton sector to have a common value $m_{\tilde{l}}$ and treat $m_{\tilde{l}}$ as a free parameter.

- Third, we assume the grand unification relation $3 M_{1} /\left(5 \alpha_{1}\right)=M_{2} / \alpha_{2}$ for electroweak gaugino masses, and set gluino mass at $2 \mathrm{TeV}$.

With above assumptions, we use the package NMSSMTools-4.0.0 [63, 64] to scan following parameter space of $\lambda$-SUSY:

$$
\begin{gathered}
0.7<\lambda \leq 2, \quad 0<\kappa \leq 2, \quad 100 \mathrm{GeV} \leq M_{A}, M_{P}, \mu \leq 3 \mathrm{TeV} \\
100 \mathrm{GeV} \leq M_{Q_{3}}, M_{U_{3}} \leq 2 \mathrm{TeV}, \quad\left|A_{t}\right| \leq 5 \mathrm{TeV} \\
1 \leq \tan \beta \leq 15, \quad 100 \mathrm{GeV} \leq m_{\tilde{l}}, M_{2} \leq 1 \mathrm{TeV}
\end{gathered}
$$

where all the parameters are defined at the scale of $1 \mathrm{TeV}$. During the scan, we keep samples that satisfy following constraints:

(1) The SM-like Higgs boson lies around $125 \mathrm{GeV}$ : $120 \mathrm{GeV} \leq m_{h} \leq 130 \mathrm{GeV}, m_{\tilde{t}_{i}} \geq$ $200 \mathrm{GeV}$ as suggested by the LHC search for stops [65-69], and also the bound on $\lambda, \kappa$ from eq. (2.11). Note that we have allowed for a rather wide range of $m_{h}$ in our analysis. This is because $\lambda$ larger than 1 may induce a sizable correction to 
$m_{h}$ at two-loop level [70], which is not considered in the NMSSMTools. We take this fact into account in following discussion by assuming a total (theoretical and experimental) uncertainty of $2.5 \mathrm{GeV}$ for $m_{h}$ in the fit to the Higgs data collected at the LHC, so the sample with $m_{h}$ deviating from $125 \mathrm{GeV}$ by $5 \mathrm{GeV}$ may still be acceptable by the data.

(2) All the constraints implemented in the package NMSSMTools-4.0.0, which are from the LEP search for sparticles (including the lower bounds on various sparticle masses and the upper bounds on the chargino/neutralino pair production rates), the $Z$-boson invisible decay, the $B$-physics observables such as the branching ratios for $B \rightarrow X_{s} \gamma$ and $B_{s} \rightarrow \mu^{+} \mu^{-}$, and the mass differences $\Delta M_{d}$ and $\Delta M_{s}$, the discrepancy of the muon anomalous magnetic moment, the dark matter relic density and the LUX limits on the scattering rate of dark matter with nucleon. In getting the constraint from a certain observable which has an experimental central value, we use its latest measured result and require the NMSSM to explain the result at $2 \sigma$ level.

(3) Constraints from the search for Higgs bosons at the LEP, the Tevatron and the LHC. We implement these constraints with the package HiggsBounds-4.0.0 [71, 72].

(4) Constraints from the stability of the scalar potential at one-loop level, including the absence of charge and color breakings [73-77]. We use the package Vevacious1.1.02 [73, 78-83] to implement the constraints by assuming that only the CP-even Higgs fields, stau fields and stop fields are possible to develop non-zero vacuum expectation values. We checked that samples with a large $A_{t} / \sqrt{M_{Q_{3}}^{2}+M_{U_{3}}^{2}}$ are disfavored by such constraints.

(5) Indirect constraints from the electroweak precision data such as $\rho_{\ell}, \sin ^{2} \theta_{\mathrm{eff}}^{\ell}, M_{W}$ and $R_{b}$. We require all these quantities in the NMSSM within the $2 \sigma$ range of their experimental values. We compute these observables with the formula presented in [54]. Note these constraints are important in limiting $\tan \beta$ in $\lambda$-SUSY $[53,54]$.

For each surviving sample, we further perform a fit to the Higgs data updated in this summer. These data include the measured signal strengthes for $\gamma \gamma, Z Z^{*}, W W^{*}, b \bar{b}$ and $\tau \bar{\tau}$ channels, and their explicit values are shown in figure 2 of [5], figure 20 of [6] and figure 20 of [7] for the ATLAS results, in figure 5 of [8] for the CMS results and in figure 15 of [84] for the $\mathrm{CDF}+\mathrm{D} 0$ results. We totally use 26 sets of experimental data with 24 of them corresponding to the measured signal strengthes and the other 2 being the combined masses of the Higgs boson reported by the ATLAS and the CMS collaborations respectively $[8,85]$. In calculating corresponding $\chi^{2}$, we use the method first introduced in $[86,87]$, consider the correlations among the data like done in [88-91], and assume an uncertainty of $m_{h}$ to be $2.5 \mathrm{GeV}$ (to estimate the Higgs mass contribution on the fit). For the surviving samples, we obtain $\chi_{\min , 2014}^{2} /$ d.o. $f=11.7 / 15$, where $\chi_{\min , 2014}^{2}$ represents the minimal value of the $\chi^{2}$ with the Higgs data in 2014, and the total number of the degree of freedom (d.o.f.) is counted in a naive way as $\nu=n_{\text {obs }}-n_{\text {para }}$ [36] with $n_{\text {obs }}=26$ denoting the set number of the experimental data and $n_{\text {para }}=11$ being the number of the model free 

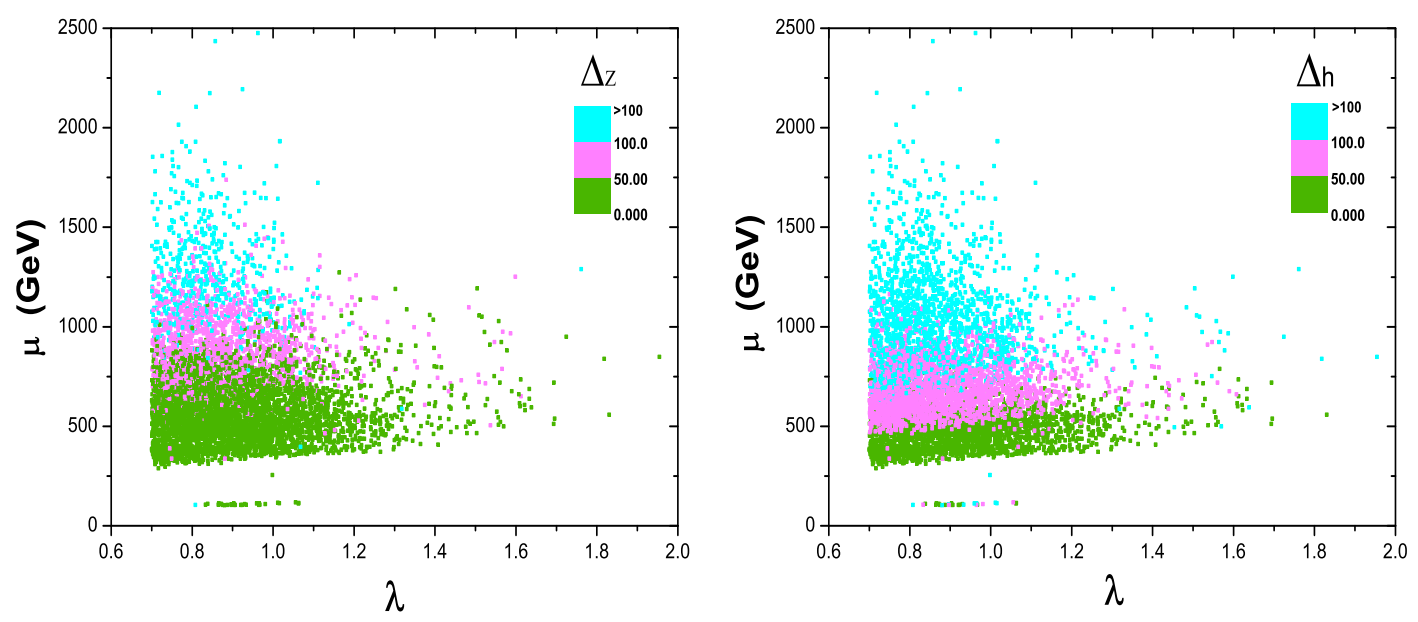

Figure 1. Samples surviving the constraints 1-3 and meanwhile satisfying $\chi^{2} \leq 25$, projected on the plane of $\mu$ versus $\lambda$. For these samples, their predictions on the fine tuning parameters $\Delta_{Z}$ and $\Delta_{h}$ are marked with different colors.

parameters listed in eq. (2.2). In the following, we concentrate on the samples satisfying $\chi^{2} \leq 25$. These samples are interpreted in statistics as the points that keep consistency with the Higgs data at $95 \%$ C.L..

Compared with the similar fit done in 2013 (see for example that in [92]), we find $\chi_{\min , 2013} \simeq 17$ for the same set of the surviving samples, which is significantly larger than $\chi_{\min , 2014}$. This reflects the more consistency of the two collaboration results in describing the properties of the discovered boson. Moreover, in order to check the validity of our new fit we also perform Higgs fits for the surviving samples by using the package HiggsSignal1.2.0 [93, 94], where 84 sets of data obtained before March 2014 are used. Similar to the new fit, this time we set the theoretical uncertainty of $m_{h}$ to be $2.5 \mathrm{GeV}$. We find that, although fewer data are employed in the new fit, the $95 \%$ C.L. constraints of the two fits on the surviving samples coincide well with each other. For example, we have totally 7015 samples surviving the constraints from items (1-5), and we find that 6553 (6577) of them further satisfy the limitation from the new fit (the fit with HiggsSignal).

At this stage, we emphasize that since the main advantage of $\lambda$-SUSY over the MSSM is its naturalness in predicting $m_{Z}$ and $m_{h}, \Delta_{Z}$ and $\Delta_{h}$ should be used as a criteria in estimating the goodness of the parameter points, that is, samples with very large $\Delta_{Z}$ and $\Delta_{h}$ should be viewed as theoretically disfavored even though they may agree well with various measurements. Numerically speaking, considering that $\Delta_{Z}$ in the MSSM are usually larger than 50 [35] (note the definition of $\Delta_{Z}$ in [35] differs from that in this work by a factor 2), we take $\max \left\{\Delta_{Z}, \Delta_{h}\right\} \leq 50$ as a standard for naturalness. To exhibit the characters of $\Delta_{Z}$ and $\Delta_{h}$ in $\lambda$-SUSY, in figure 1 we project the surviving samples on the plane of $\mu$ versus $\lambda$ with their corresponding values of $\Delta_{Z}$ and $\Delta_{h}$ marked with different colors. This figure indicates that the samples with relatively low $\Delta_{Z}$ and $\Delta_{h}$ are characterized by low values of $\mu$, or numerically speaking, requiring $\max \left\{\Delta_{Z}, \Delta_{h}\right\} \leq 50$ results in $\mu \lesssim 780 \mathrm{GeV}$. This can be intuitively understood by the fact that $\mu=\lambda v_{s}$ with 

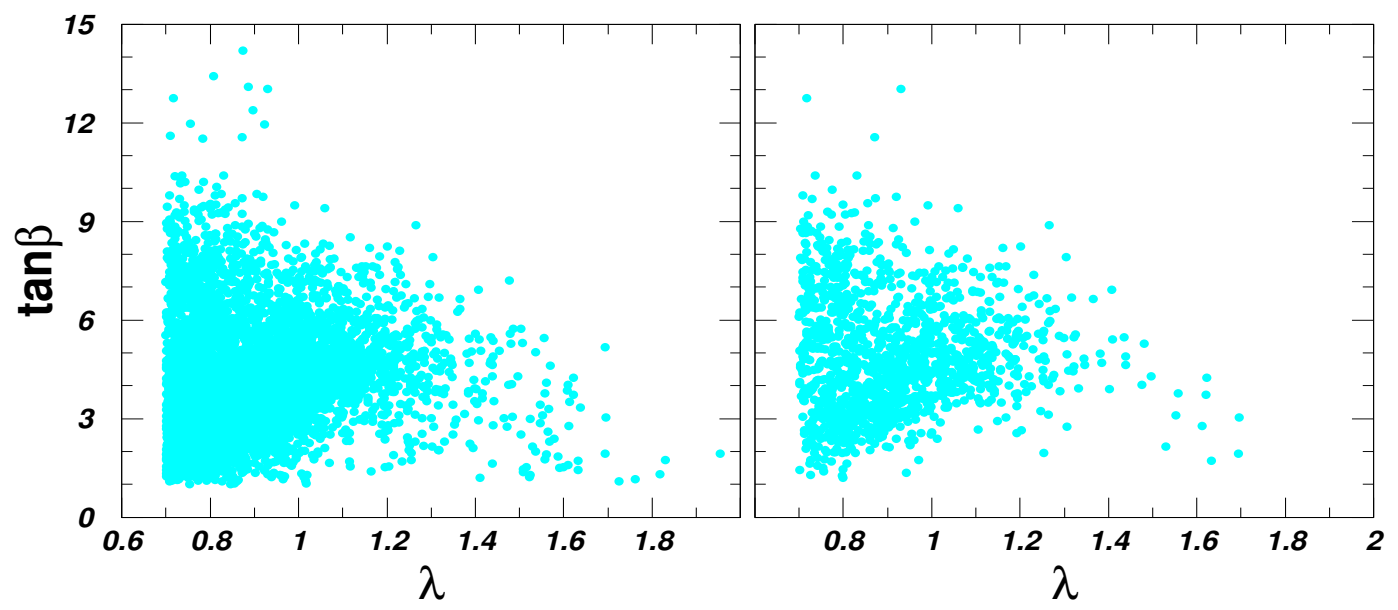

Figure 2. Surviving samples projected on $\tan \beta-\lambda$ plane. Samples in the left panel are same as that of figure 1, while samples in the right panel are further required to satisfy $\max \left\{\Delta_{Z}, \Delta_{h}\right\} \leq 50$.

the natural size of $v_{s}$ lying at the weak scale. Furthermore, we checked that $\Delta_{Z}$ and $\Delta_{h}$ are more sensitive to $\lambda$ than to the other SUSY parameters for most of the surviving samples.

Since $\lambda$ and $\tan \beta$ are two most important parameters in $\lambda$-SUSY, we pay particular attention to their correlation. In figure 2 we show all the surviving samples on the $\tan \beta-\lambda$ plane without and with the requirement $\max \left\{\Delta_{Z}, \Delta_{h}\right\} \leq 50$ (see left panel and right panel respectively). This figure indicates that $\tan \beta$ tends to decrease with the increase of $\lambda$, and for $\lambda>1, \tan \beta \leq 10$. The main reason for such a behavior is, as we mentioned before, due to the constraints from the electroweak precision data. This figure also indicates that after requiring $\max \left\{\Delta_{Z}, \Delta_{h}\right\} \leq 50$, a large portion of samples with relatively low values of $\tan \beta$ are excluded. The reason is that $m_{h, \text { tree }}^{2}$ in $\lambda$-SUSY is usually larger than $125 \mathrm{GeV}$ and a high value of $\tan \beta$ is able to reduce the value of $m_{h, \text { tree }}^{2}$.

Based on above arguments and meanwhile in order to show the preference of the Higgs data and the fine tuning argument on the parameter space, we classify the surviving samples into three types as follows:

- Type-I samples: those with $\chi^{2} \leq 25$ and meanwhile $\max \left\{\Delta_{Z}, \Delta_{h}\right\} \leq 50$. This type of sample is considered as the physical sample in our discussion.

- Type-II samples: those with $\chi^{2} \leq 25$ but $\max \left\{\Delta_{Z}, \Delta_{h}\right\}>50$. This type of sample can not be excluded by experiments, but is not favored by the fine tuning argument.

- Type-III samples: those with $\chi^{2}>25$. Obviously, this type of sample is of less interest than the previous two types.

For completeness, we present in table 1 the allowed ranges for these samples. As shown in figure 1 and figure 2 and also in this table, with the increase of $\lambda$ the parameter space of $\lambda$-SUSY are crushed into a narrow region until $\lambda$ reaches its maximum, which is about 1.8. 


\begin{tabular}{|c|c|c|c|}
\hline Parameters & Type-I Samples & Type-(I+II) Samples & Type-(I+II+III) Samples \\
\hline$\lambda$ & $0.7 \sim 1.8$ & $0.7 \sim 1.9$ & $0.7 \sim 2$ \\
\hline$\kappa$ & $0.2 \sim 1.9$ & $0.1 \sim 1.9$ & $0.1 \sim 2.0$ \\
\hline $\tan \beta$ & $1.2 \sim 14.2$ & $1 \sim 14.2$ & $1 \sim 15$ \\
\hline$\mu(\mathrm{GeV})$ & $105 \sim 870$ & $105 \sim 2700$ & $100 \sim 2200$ \\
\hline$M_{A}(\mathrm{GeV})$ & $365 \sim 3000$ & $345 \sim 3000$ & $340 \sim 3000$ \\
\hline$M_{P}(\mathrm{GeV})$ & $65 \sim 3000$ & $60 \sim 3000$ & $20 \sim 3000$ \\
\hline$M_{1}(\mathrm{GeV})$ & $50 \sim 470$ & $50 \sim 500$ & $50 \sim 500$ \\
\hline$M_{Q_{3}}(\mathrm{GeV})$ & $200 \sim 2000$ & $200 \sim 2000$ & $200 \sim 2000$ \\
\hline$M_{U_{3}}(\mathrm{GeV})$ & $200 \sim 2000$ & $200 \sim 2000$ & $200 \sim 2000$ \\
\hline$A_{t}(\mathrm{GeV})$ & $-4500 \sim 4300$ & $-5000 \sim 4800$ & $-5000 \sim 5000$ \\
\hline$M_{\tilde{l}}(\mathrm{GeV})$ & $100 \sim 620$ & $100 \sim 620$ & $100 \sim 750$ \\
\hline$A_{\lambda}(\mathrm{GeV})$ & $-1100 \sim 2900$ & $-3200 \sim 2900$ & $-2800 \sim 3000$ \\
\hline$A_{\kappa}(\mathrm{GeV})$ & $-2600 \sim 180$ & $-2600 \sim 200$ & $-2600 \sim 450$ \\
\hline
\end{tabular}

Table 1. Allowed ranges for different parameters. All types of samples survive the constraints 1-3, and they differ only by their predictions on $\chi^{2}$ and $\max \left\{\Delta_{Z}, \Delta_{h}\right\}$ (see their definitions at the end of subsection B).

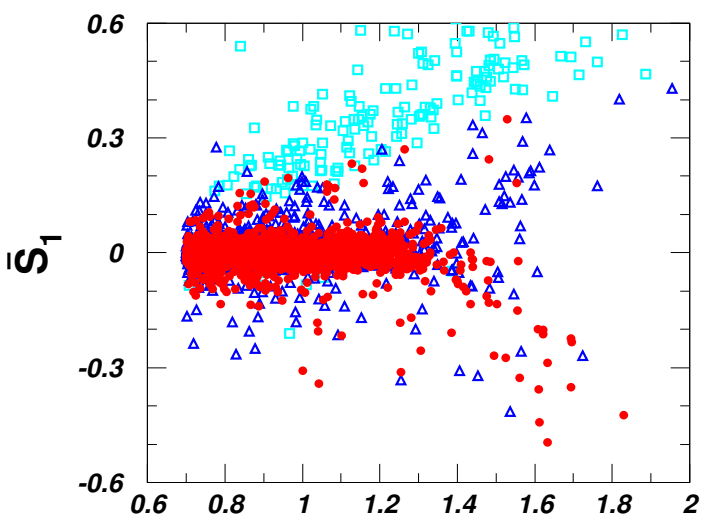

$\lambda$

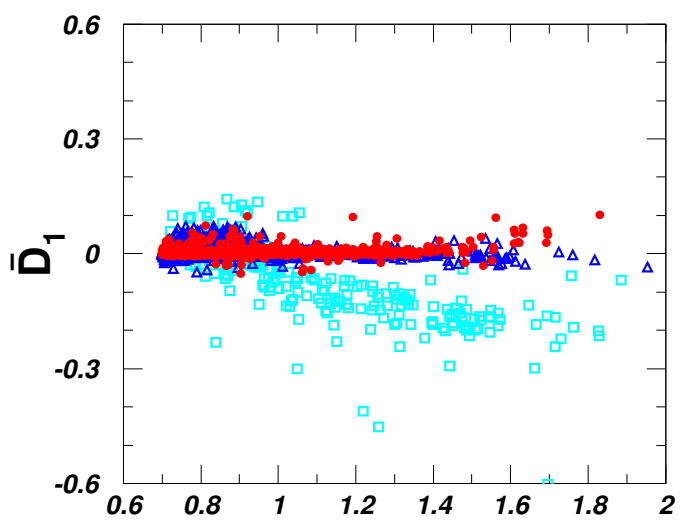

$\lambda$

Figure 3. Singlet component coefficient $\bar{S}_{1}$ and non-SM doublet component coefficient $\bar{D}_{1}$ of the SM-like Higgs boson as a function of $\lambda$. Here red bullet, blue triangle and sky-blue square denote Type-I sample, Type-II sample and Type-II sample respectively.

\section{Properties of $h_{1}$ and $h_{2}$}

In this section, we explore the Higgs sector of $\lambda$-SUSY to exhibit the properties of the lightest and the next-to-lightest CP-even Higgs bosons. We pay particular attention to the features of the bosons that differentiate $\lambda$-SUSY from the MSSM or from the NMSSM with a low $\lambda$. 

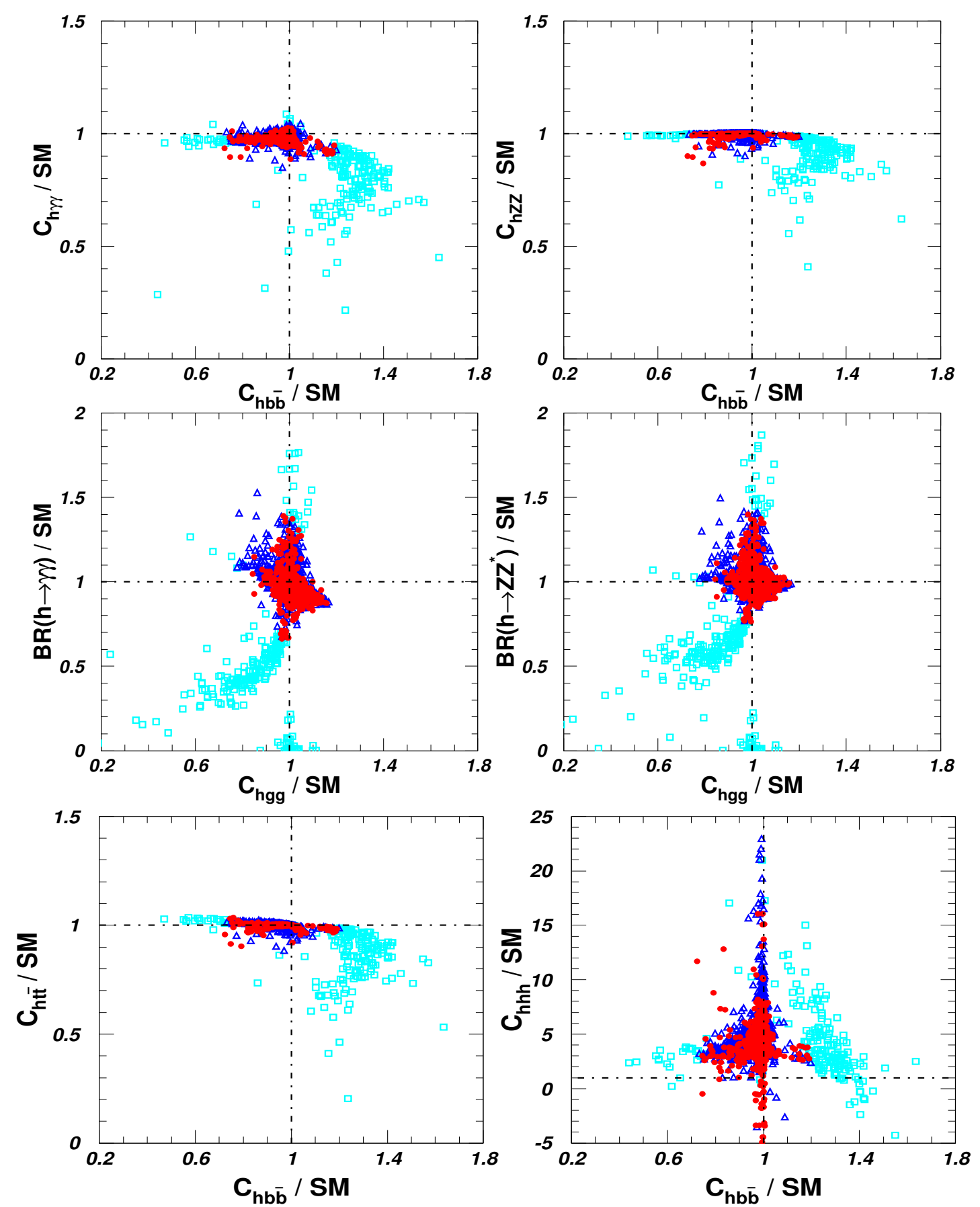

Figure 4. Coupling information of the SM-like Higgs boson for Type-I sample (red bullet), Type-II sample (blue triangle) and Type-III sample (sky-blue square).

\subsection{Properties of the lightest CP-even Higgs boson}

As we mentioned before, throughout this work we treat the lightest CP-even Higgs boson as the SM-like Higgs boson, so some properties of $h$ such as its coupling to vector bosons have been limited to closely mimic those of the SM Higgs boson. However, as we will show 
below, the triple self coupling of the boson or more general the Higgs potential may still differ greatly from that of the SM.

In figure 3 , we show the singlet component coefficient $\bar{S}_{1}$ and non-SM doublet component coefficient $\bar{D}_{1}$ of $h$ for Type-I samples (red bullet), Type-II samples (blue triangle) and Type-III samples (sky-blue square) on the left panel and right panel respectively. This figure indicates that $\left|\bar{S}_{1}\right|$ may exceed 0.6 without considering the Higgs data, and it is up bounded by about 0.5 at $95 \%$ C.L. after considering the data. In contrast, $\left|\bar{D}_{1}\right|$ reaches at most about 0.3 and 0.1 before and after considering the data respectively, and given $\bar{S}_{1} \neq 0$, we find it is always much smaller than $\left|\bar{S}_{1}\right|$ after considering the data. The reason for the difference between $\bar{S}_{1}$ and $\bar{D}_{1}$ is that the constraints we considered have put nontrivial requirements on the elements $\mathcal{M}_{11}^{2}$ and $\mathcal{M}_{12}^{2}$ of the CP-even Higgs mass matrix, e.g. $\mathcal{M}_{11}^{2} \gtrsim 200 \mathrm{GeV}^{2}$ and $\left|\mathcal{M}_{12}^{2} / \mathcal{M}_{11}^{2}\right| \ll 1$, so $\left|\bar{D}_{1}\right|$ is forbidden to be moderately large. In comparison, $\mathcal{M}_{33}^{2}$ is less constrained due to the singlet nature of the field $S_{3}$, and given $\mathcal{M}_{33}^{2} \simeq \mathcal{M}_{22}^{2},\left|\bar{S}_{1}\right|$ may be as large as 0.7 . Furthermore, from the coupling expressions of $h$ in eq. (2.9) one can learn that the $h Z Z$ coupling is always suppressed in comparison with its SM value due to the non-vanishing of $\bar{S}_{1}$ and $\bar{D}_{1}$, while for the fermion Yukawa couplings $Y_{h \bar{f} f}$, depending the sign of $\bar{D}_{1}$ it may be either enhanced or suppressed. Explicitly speaking, given $\bar{S}_{1}=0$ and $\left|\bar{D}_{1}\right|<0.1$, one can learn that $C_{h \bar{u} u}$ is slightly enhanced while $C_{h \bar{d} d}$ is suppressed if $\bar{D}_{1}$ is positive, and the situation reverses for the couplings if $\bar{D}_{1}$ changes its sign. In any case, the larger $\left|\bar{S}_{1}\right|$ becomes, the smaller the couplings are. Figure 3 also indicates that the values of $\left|\bar{S}_{1}\right|$ and $\left|\bar{D}_{1}\right|$ tend to increase with the increase of $\lambda$, and so are the deviations of the normalized couplings from unity. The reason is that $m_{h \text {,tree }}$ will be much larger than $125 \mathrm{GeV}$ for a sufficient large $\lambda$, and sizable mixings must intervene to pull down the mass.

We also compare our results in figure 3 with those in [48], where a similar fit was performed by using the Higgs data in 2013 in the framework of $\lambda$-SUSY. We find that now the allowed ranges of $\left|\bar{S}_{1}\right|$ and $\left|\bar{D}_{1}\right|$ shrink significantly. This reflects the more tightness of the constraints we considered in limiting the Higgs properties.

Now let's turn to the couplings of $h$. In figure 4 , we exhibit such information for same samples as those in figure 3. This figure indicates that after imposing the constraints from the Higgs data, the normalized couplings $C_{h \gamma \gamma} / S M, C_{h Z Z} / S M$ and $C_{h \bar{t} t} / S M$ are limited within $15 \%$ deviation from unity, and the couplings $C_{h g g} / S M$ and $C_{h \bar{b} b} / S M$ are at most $25 \%$ and $40 \%$ deviating from unity respectively. Moreover, due to the change of the width of $h$ which is mainly determined by $C_{h \bar{b} b}$, the normalized branching ratios $\operatorname{Br}(h \rightarrow \gamma \gamma) / S M$ and $\operatorname{Br}\left(h \rightarrow Z Z^{*}\right) / S M$ may vary from 0.6 to 1.5 . Compared with the similar fit results in 2012 [35], we find that the optimal values of the couplings are now shifted significantly.

Maybe the most impressive feature of $h$ in $\lambda$-SUSY is that the strength of its triple self coupling $C_{h h h} / S M$ may get enhanced by a factor over 10. This is shown on the right panel of the third row in figure 4, which exhibits that $C_{h h h} / S M$ may reach 16 and 23 for the Type-I samples and Type-II samples respectively. Here we remind that that such a great enhancement can not occur in the MSSM where the quartic terms of the Higgs potential are determined by the weak coupling [95]. We also remind that the enhancement seems to be limited by the naturalness argument. To see this, we list two benchmark points 


\begin{tabular}{|c|c|c|c|c|}
\hline No. of Point & Point 1 (P1) & Point 2 (P2) & Point 3 (P3) & Point 4 (P4) \\
\hline$\lambda$ & 0.86 & 0.94 & 0.75 & 0.71 \\
$\kappa$ & 1.27 & 1.19 & 1.64 & 1.64 \\
$\tan \beta$ & 1.59 & 1.82 & 1.45 & 1.35 \\
$\mu(\mathrm{GeV})$ & 697.6 & 1100.7 & 614.0 & 599.8 \\
$M_{A}(\mathrm{GeV})$ & 2127.9 & 2237.9 & 388.1 & 372.6 \\
$M_{P}(\mathrm{GeV})$ & 1449.4 & 283.8 & 2282.4 & 2135.2 \\
$M_{1}(\mathrm{GeV})$ & 169.9 & 200.7 & 83.2 & 70.5 \\
$M_{Q_{3}}(\mathrm{GeV})$ & 1646.3 & 675.5 & 409.2 & 1587.8 \\
$M_{U_{3}}(\mathrm{GeV})$ & 511.0 & 1435.6 & 697.6 & 894.7 \\
$M_{\tilde{l}}(\mathrm{GeV})$ & 186.5 & 218.5 & 128.4 & 112.4 \\
$A_{t}(\mathrm{GeV})$ & -25.1 & 1539.6 & 881.1 & -1639.8 \\
$A_{\lambda}(\mathrm{GeV})$ & 766.0 & -310.5 & -1248.9 & -1302.0 \\
$A_{\kappa}(\mathrm{GeV})$ & -674.5 & -12.5 & -1602.3 & -1032.6 \\
\hline$m_{h}(\mathrm{GeV})$ & 125.0 & 125.4 & 125.2 & 124.4 \\
$m_{H_{2}}(\mathrm{GeV})$ & 1697.3 & 2144.6 & 232.4 & 245.0 \\
$m_{A_{1}}(\mathrm{GeV})$ & 1440.5 & 174.0 & 150.5 & 102.3 \\
$m_{H^{ \pm}}(\mathrm{GeV})$ & 2088.2 & 2153.7 & 228.1 & 238.6 \\
$\bar{D}_{1}$ & $-8 \times 10^{-5}$ & $2 \times 10^{-3}$ & 0.01 & 0.04 \\
$\bar{S}_{1}$ & 0.02 & -0.02 & $6 \times 10^{-3}$ & $-3 \times 10^{-3}$ \\
$\bar{D}_{2}$ & -0.22 & -0.98 & -0.99 & -0.99 \\
$\bar{D}_{A_{1}}$ & 0.08 & 0.10 & 0.99 & 0.99 \\
\hline$\chi^{2}$ & 12.2 & 12.1 & 12.0 & 13.5 \\
$\Delta_{Z}$ & 35.4 & 139.6 & 46.9 & 45.1 \\
$\Delta_{h}$ & 38.4 & 233.2 & 89.5 & 89.1 \\
$C_{h h h} / S M$ & 13.7 & 22.1 & 5.1 & 4.2 \\
$\sigma(g g \rightarrow h h) / S M$ & 34.3 & 96.1 & 1.3 & 0.6 \\
\hline
\end{tabular}

Table 2. Benchmark points for different cases considered in this work. Note all the input parameters are defined at $1 \mathrm{TeV}$, and in calculating the spectrum of the Higgs bosons, important radiative corrections have been taken into account.

with large $C_{h h h}$ in table 2 (see points P1 and P2). One can easily learn that each point corresponds to a low Higgs $\chi^{2}$ and meanwhile a relative large $\Delta_{Z}$ and $\Delta_{h}$, indicating that naturalness disfavors a too large $C_{h h h}$ in $\lambda$-SUSY.

\subsection{Properties of the next-to-lightest CP-even Higgs boson}

Considering that $h_{3}$ in $\lambda$-SUSY is usually at $\mathrm{TeV}$ scale and thus it decouples from the electroweak physics, we here only study the property of the Next-to-Lightest CP-even Higgs boson $h_{2}$. As we will show below, such a study is helpful to understand the Higgs pair production process.

In figure 5, we show the non-SM doublet component $\bar{D}_{2}^{2}$ as a function of $m_{h_{2}}$ for Type-I, Type-II and Type-III samples. This figure reveals following information: 


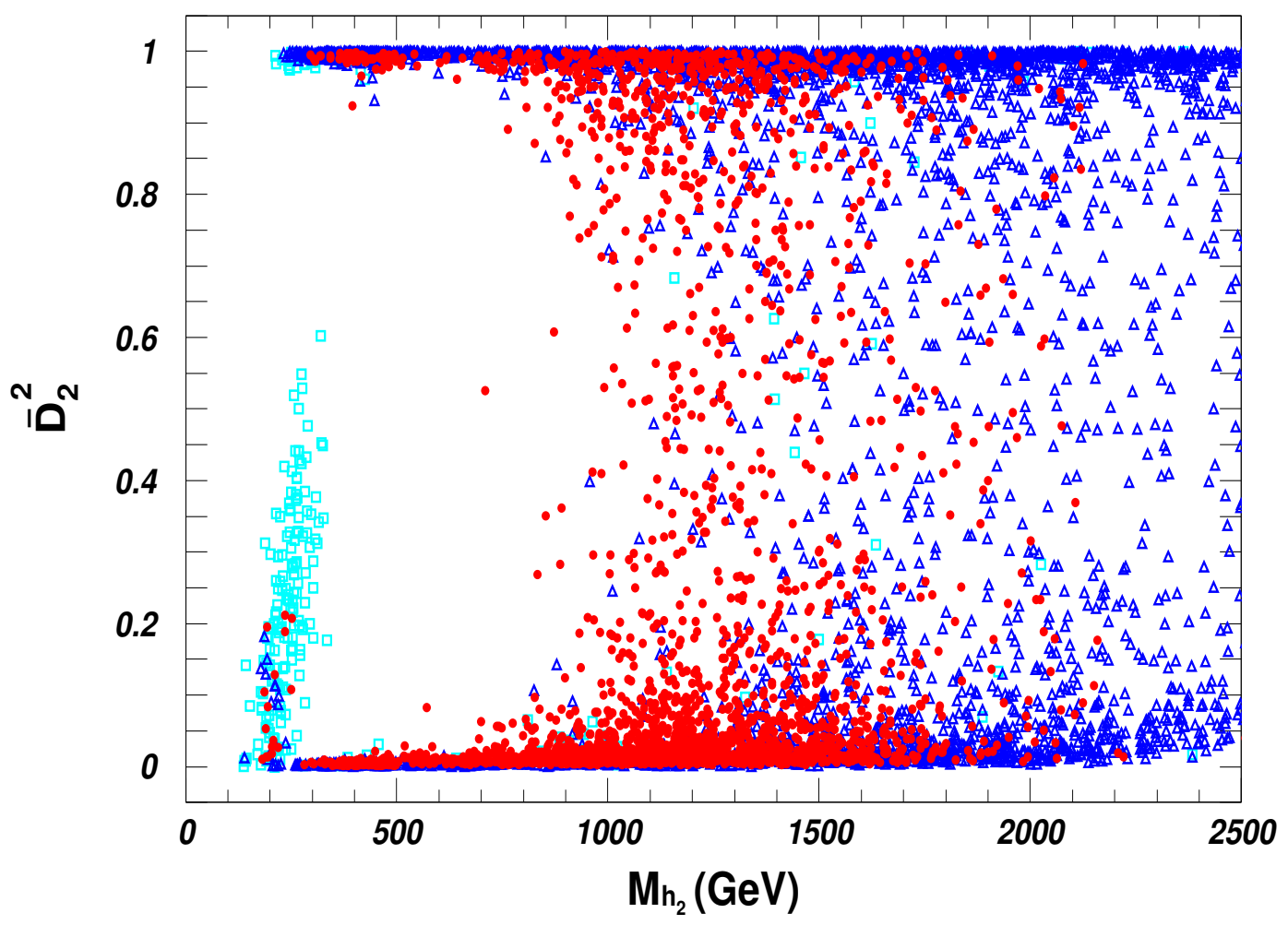

Figure 5. Doublet component of $h_{2}$ as a function of $m_{h_{2}}$ for the same samples as figure 3. Again, Type-I, Type-II and Type-III samples are marked with red bullet, blue triangle and sky-blue square, respectively.
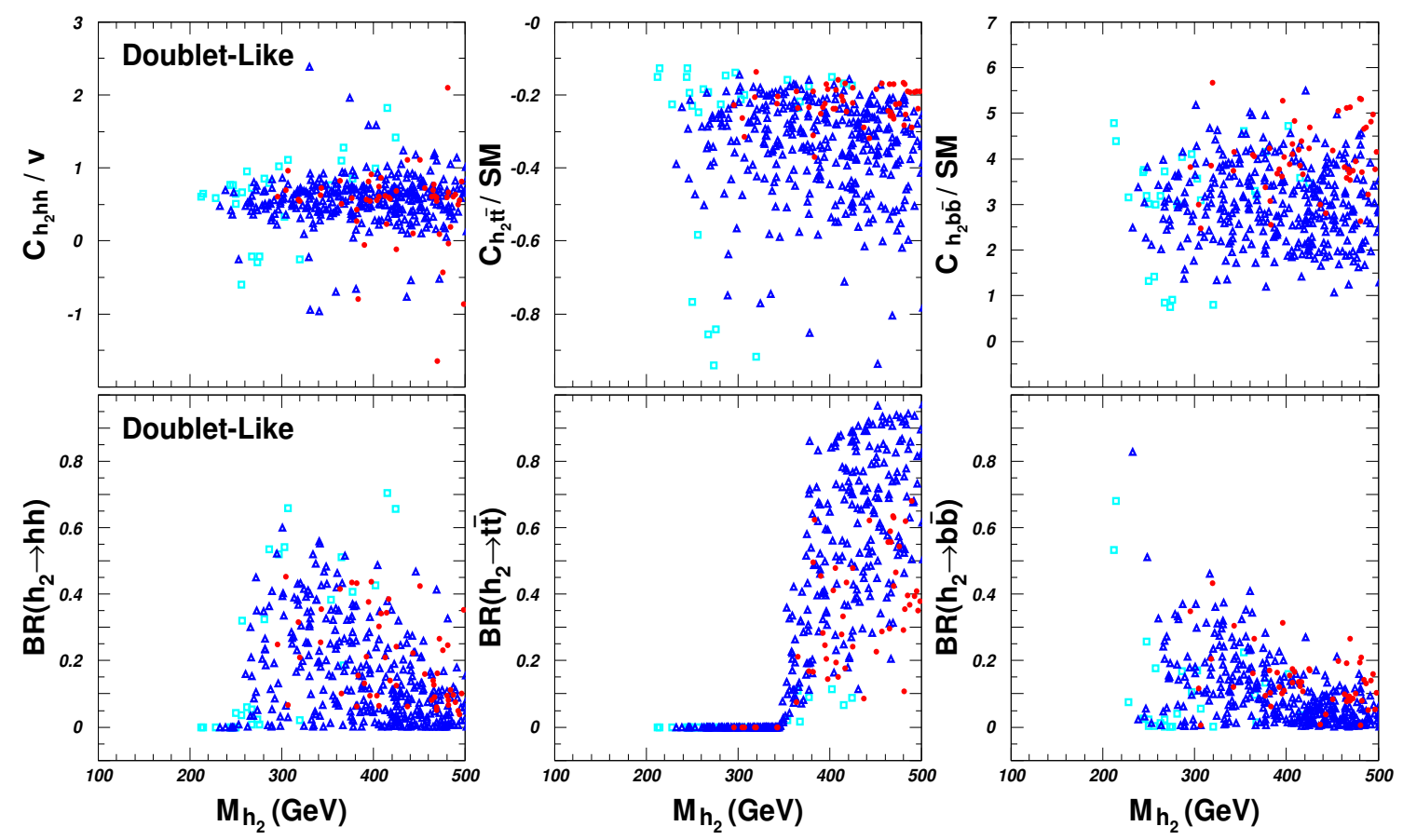

Figure 6. Couplings and Branching ratios of $h_{2}$ as a function of $h_{2}$. Note that only the samples with doublet dominated $h_{2}$ in figure 6 are considered. 
- For $m_{h_{2}} \leq 500 \mathrm{GeV}, \bar{D}_{2}^{2}$ is either around 1 or around 0 for most Type-I and Type-II samples. In this case, the mixing between the fields $S_{1}$ and $S_{3}$ is small in forming $h_{2}$, which can be obtained if $\left|\mathcal{M}_{13}^{2}\right| \ll\left|\mathcal{M}_{11}^{2}-\mathcal{M}_{33}^{2}\right|$. Note such a situation is not altered until $m_{h_{2}} \gtrsim 700 \mathrm{GeV}$.

- In case of $\bar{D}_{2}^{2} \simeq 1, h_{2}$ is obviously non-SM doublet dominated, while in case of $\bar{D}_{2}^{2} \simeq 0$ $h_{2}$ should be singlet dominated since $\bar{D}_{3}^{2}=1-\bar{D}_{1}^{2}-\bar{D}_{2}^{2} \simeq 1$, which implies that $h_{3}$ is non-SM doublet dominated.

- The doublet dominated $h_{2}$ can be as light as $200 \mathrm{GeV}$, which is quite different from the situation of the MSSM where the non-SM Higgs boson $H$ must be heavier than about $300 \mathrm{GeV}$ after considering various constraints [96]. As a comparison, the singlet dominated $h_{2}$ is more loosely limited so that it can be lighter than $150 \mathrm{GeV}$.

- Note for $m_{h_{2}} \lesssim 300 \mathrm{GeV}$, there exist some type-III samples with $\bar{D}_{2}^{2}>0.2$. Since $\bar{D}_{3}^{2}=1-\bar{D}_{1}^{2}-\bar{D}_{2}^{2}<0.8$, these samples predict a $h_{3}$ with sizable singlet and/or SM doublet components. This mixing pattern can be achieved only for a not too heavy $h_{3}$. In fact, we examined the properties of these sample, and found $m_{h_{3}} \lesssim 650 \mathrm{GeV}$ and $\mu \lesssim 200 \mathrm{GeV}$. Since all CP-even Higgs bosons in this case are relatively light, it is apt to be tightly limited by the Higgs data.

- Naturalness should play a role in limiting the properties of $h_{2}$ [97]. Explicitly speaking, figure 5 shows that there are few Type-I samples with $m_{h_{2}}>2000 \mathrm{GeV}$, which may be interpreted as that naturalness prefers a relatively light $h_{2}$. Another example is the fraction of Type-I samples in the total number of Type-I plus Type-II samples for the doublet dominated $h_{2}$ is significantly lower than that for the singlet dominated $h_{2}$, which means that naturalness tends to put a tighter constraint on the doublet dominated $h_{2}$. All these features can be intuitively understood by the fact that since $v_{u}, v_{d} \sim 100 \mathrm{GeV}$, a too heavy non-SM doublet dominated or singlet dominated $h_{2}$ will make the theory fine tuned to get the correct electroweak symmetry breaking.

In the following, we try to illustrate the properties of $h_{2}$ for Type-I and Type-II samples with $m_{h_{2}} \leq 500 \mathrm{GeV}$. Most of these samples are characterized by either $\bar{D}_{2}^{2} \simeq 1$ or $\bar{D}_{2}^{2} \simeq 0$, which is very helpful to simplify our analysis.

We first concentrate on a doublet dominated $h_{2}$. Since $\bar{D}_{2}^{2} \simeq 1$, the couplings of the $h_{2}$ can be approximated by:

$$
C_{h_{2} V V} / S M \simeq 0, \quad C_{h_{2} \bar{u} u} / S M \simeq \operatorname{Sign}\left(\bar{D}_{2}\right) \cot \beta, \quad C_{h_{2} \bar{d} d} / S M \simeq-\operatorname{Sign}\left(\bar{D}_{2}\right) \tan \beta .(3
$$

In figure 6 , we only consider the doublet dominated $h_{2}$ in figure 5 and show their normalized couplings such as $C_{h_{2} \bar{t} t} / S M, C_{h_{2} \bar{b} b} / S M$ and $C_{h_{2} h h} / v$ as functions of $m_{h_{2}}$. We also plot the branching ratios of $h_{2} \rightarrow \bar{t} t, h_{2} \rightarrow \bar{b} b$ and $h_{2} \rightarrow h h$ in a similar way.

From figure 6, we can learn following features about Type-I and Type-II samples:

- In most cases, eq. (3.1) is a good approximation for the three $h_{2}$ couplings, especially for the coupling $C_{h_{2} \bar{b} b} / S M$. 
- In general, with the increase of $m_{h_{2}}$ the couplings $C_{h_{2} \bar{t} t} / S M$ and $C_{h_{2} \bar{b} b} / S M$ may vary within a wider ranges. This is because the constraints we considered get relaxed as $h_{2}$ becomes heavy so that the couplings become more flexible to satisfy the constraints. This character also applies to the singlet dominated $h_{2}$.

- $\left|C_{h_{2} \bar{t} t} / S M\right|$ is not too small: $\left|C_{h_{2} \bar{t} t} / S M\right| \gtrsim 0.2$, and in optimal case, it is just slightly below 1 . On the other hand, $\left|C_{h_{2}} \bar{b} b / S M\right|$ is usually larger than 1 with its maximum value reaching 6 . In this case, the $h_{2} g g$ coupling is given by

$$
\begin{aligned}
\left|\frac{C_{h_{2} g g}}{C_{h g g}^{\mathrm{SM}}}\right| & \simeq \frac{\cot \beta A_{\frac{1}{2}}\left(\frac{m_{h_{2}}^{2}}{4 m_{t}^{2}}\right)-\tan \beta A_{\frac{1}{2}}\left(\frac{m_{h_{2}}^{2}}{4 m_{t}^{2}}\right)}{A_{\frac{1}{2}}\left(\frac{m_{h}^{2}}{4 m_{t}^{2}}\right)} \\
& \simeq \begin{cases}\{1.5 \cot \beta-(-0.03+0.03 i) \tan \beta\} / 1.4 & \text { for } m_{h_{2}}=250 \mathrm{GeV}, \\
\{(2.0+0.01 i) \cot \beta-(-0.02+0.02 i) \tan \beta\} / 1.4 & \text { for } m_{h_{2}}=350 \mathrm{GeV}, \\
\{(2.1+1.1 i) \cot \beta-(-0.01+0.01 i) \tan \beta\} / 1.4 & \text { for } m_{h_{2}}=450 \mathrm{GeV}, \\
\{(1.5+1.6 i) \cot \beta-(-0.01+0.01 i) \tan \beta\} / 1.4 & \text { for } m_{h_{2}}=550 \mathrm{GeV},\end{cases}
\end{aligned}
$$

where the loop function $A_{\frac{1}{2}}$ is defined in [95] and we have neglected the minor important squark contribution. This expression indicates that due to the opposite sign of the two couplings, the real parts of the top and bottom contributions to the $h_{2} g g$ interaction interfere constructively, while the imaginary parts interfere destructively, and the $h_{2} g g$ coupling strength is maximized at low $\tan \beta$.

- The potentially important decay modes of $h_{2}$ include $h_{2} \rightarrow \bar{t} t, \bar{b} b, A_{1} A_{1}, \overline{\tilde{\chi}}_{i} \tilde{\chi}_{j}$, where $A_{1}$ denotes the lighter CP-odd Higgs boson and $\tilde{\chi}_{i}$ represents a supersymmetric particle such as a neutralino. We find that $h_{2} \rightarrow t \bar{t}$ is usually the main decay mode for $m_{h_{2}} \gtrsim 400 \mathrm{GeV}$, and $h_{2} \rightarrow h h$ (any of the decays $h_{2} \rightarrow b \bar{b}, A_{1} A_{1}, \overline{\tilde{\chi}}_{i} \tilde{\chi}_{j}$ ) may be dominant over the other channels for $260 \mathrm{Gev} \lesssim m_{h_{2}} \lesssim 400 \mathrm{GeV}\left(m_{h_{2}} \lesssim 250 \mathrm{GeV}\right)$.

- Considering that the case of $m_{h_{2}} \lesssim 250 \mathrm{GeV}$ was scarcely studied before, we pay particular attention to its features. We find that the Higgs sector in this case usually exhibits an inverted mass hierarchy, i.e. the spectrum is characterized by $m_{h_{2}} \simeq$ $m_{H^{ \pm}}>m_{A_{1}}$ instead of the usual order $m_{A_{1}}>m_{H^{ \pm}}$. The underlying reason for such an anomaly is owe to the hierarchy structure of the CP-odd Higgs mass matrix in the basis $\left(P_{1}, P_{2}\right):\left|\mathcal{M}_{P, 11}^{2}\right| \ll\left|\mathcal{M}_{P, 12}^{2}\right| \ll\left|\mathcal{M}_{P, 22}^{2}\right|$. For such a mass matrix, the physical scalar $A_{1}$ can be tuned to be very light by choosing an appropriate value of $\mathcal{M}_{P, 12}^{2}$. In table 2, we list two such points (denoted by P3 and P4 respectively) with P4 further satisfying $m_{h_{2}}>2 m_{A_{1}}$.

Next we consider the singlet dominated $h_{2}$. In this case, since $\bar{D}_{2}^{2} \simeq 0$ we have

$$
1-\bar{S}_{2}^{2}-\bar{D}_{2}^{2} \simeq 1-\bar{S}_{2}^{2} \simeq \bar{S}_{1}^{2}+\bar{S}_{3}^{2},
$$

where the sum rule $\bar{S}_{2}^{2}=1-\bar{S}_{1}^{2}-\bar{S}_{3}^{2}$ is used. On the other hand, because

$$
\bar{S}_{3}^{2}+\bar{D}_{3}^{2}=\bar{S}_{3}^{2}+1-\bar{D}_{2}^{2}-\bar{D}_{1}^{2} \simeq \bar{S}_{3}^{2}+1-\bar{D}_{1}^{2} \leq 1,
$$



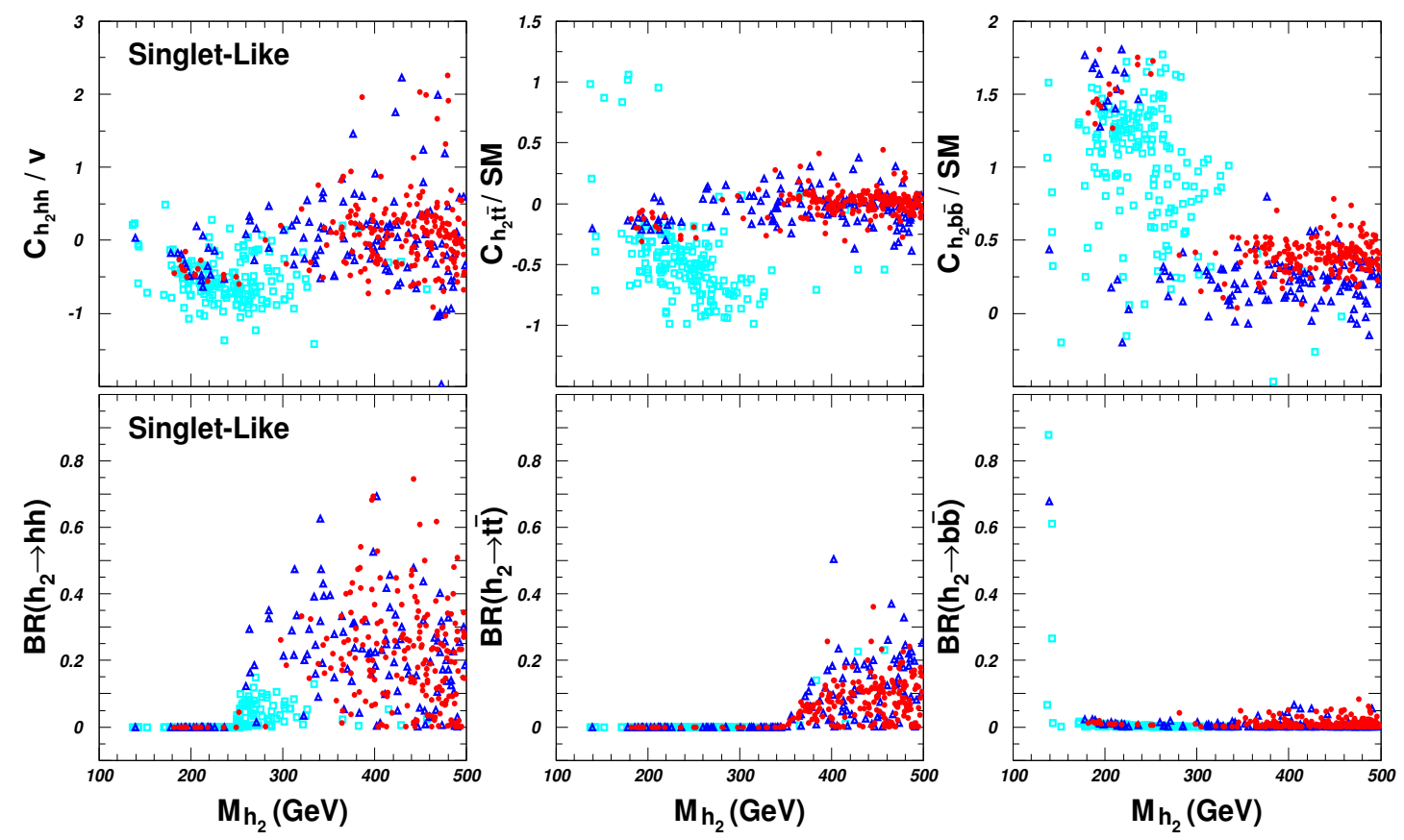

Figure 7. Same as figure 6, but for a singlet dominated $h_{2}$.
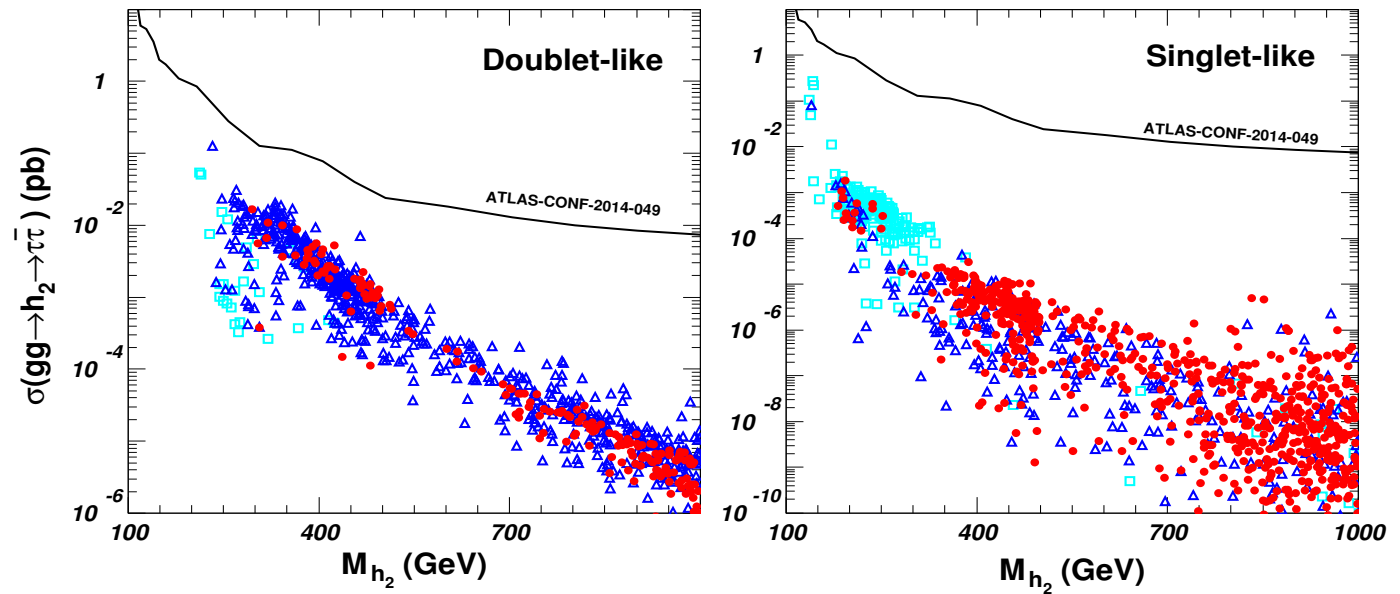

Figure 8. Same as figure 6 (left panel) and figure 7 (right panel), but showing the $\bar{\tau} \tau$ signal rates induced by the process $g g \rightarrow h_{2} \rightarrow \bar{\tau} \tau$ at 8-TeV LHC. As a comparison, the bounds from the direct search for $\bar{\tau} \tau$ signal by ATLAS collaboration are also shown.

we get

$$
\bar{S}_{3}^{2} \lesssim \bar{D}_{1}^{2}
$$

Taking eq. (3.2) and eq. (3.3) in mind, and noticing the fact that $\bar{D}_{1}^{2} \ll \bar{S}_{1}^{2}$ for a sizable $\bar{S}_{1}$ (see discussion about figure 3 ), we finally conclude that

$$
1-\bar{S}_{2}^{2}-\bar{D}_{2}^{2} \simeq \bar{S}_{1}^{2} .
$$

With this approximation, we can write down the couplings of the singlet dominated $h_{2}$ as:

$$
\begin{aligned}
C_{h_{2} V V} / S M & \simeq\left|\bar{S}_{1}\right|, \quad C_{h_{2} \bar{u} u} / S M \simeq \operatorname{Sign}\left(V_{22}\right)\left|\bar{S}_{1}\right|, \\
C_{h_{2} \bar{d} d} / S M & \simeq-\bar{D}_{2} \tan \beta+\operatorname{Sign}\left(V_{22}\right)\left|\bar{S}_{1}\right| .
\end{aligned}
$$


In figure 7, we show the couplings $C_{h_{2} \bar{t} t} / S M, C_{h_{2} \bar{b} b} / S M$ and $C_{h_{2} h h} / v$, and also the branching ratios of $h_{2} \rightarrow \bar{t} t, h_{2} \rightarrow \bar{b} b$ and $h_{2} \rightarrow h h$ in a way similar to figure 6 . This figure indicates that as suggested by above approximations, both the $h_{2} \bar{t} t$ and $h_{2} \bar{b} b$ couplings for a singletlike $h_{2}$ are usually small, but the coupling $h_{2} h h$ may still be large with $C_{h_{2} h h} / v$ reaching about 2.5 in optimal case. As a result of such couplings and meanwhile the relatively strong interaction of the $h_{2}$ with sparticles, ${ }^{2} h_{2} \rightarrow \bar{t} t$ is no longer the dominant decay channel of $h_{2}$ even for $m_{h_{2}} \gtrsim 400 \mathrm{GeV}$, instead any of $h_{2} \rightarrow h h, A_{1} A_{1}, \overline{\tilde{\chi}}_{i} \tilde{\chi}_{j}$ may become dominant once the kinematics is accessible. We checked that $h_{2} \rightarrow \overline{\tilde{\chi}}_{i} \tilde{\chi}_{j}$ is usually the main decay mode for $m_{h_{2}}<250 \mathrm{GeV}$.

In order to further show the difference between a doublet dominated $h_{2}$ and a singlet dominated $h_{2}$, we plot the rate of the $\bar{\tau} \tau$ signal induced by the process $g g \rightarrow h_{2} \rightarrow \bar{\tau} \tau$ at 8 - TeV LHC with the same samples as those in figure 6 and figure 7 respectively. For comparison, we also show the direct search bound on this signal from the recent ATLAS analysis. This figure indicates that the $\bar{\tau} \tau$ signal rate induced by a doublet dominated $h_{2}$ is usually two order larger than that by a singlet dominated $h_{2}$ with same mass, and in either case the rate is at least one order lower than the direct search bound. This means that indirect experimental constraints such as $B \rightarrow X_{s} \gamma$, the dark matter direct search result and the Higgs data play an important role in deciding the lower mass bound of $h_{2}$.

\section{Higgs pair production at the LHC}

After the discovery of the Higgs boson, the next important task of the LHC is to reconstruct the Higgs potential and finally decipher the mechanism of the electroweak symmetry breaking. In this direction, the Higgs pair production plays an unique role since it involves the Higgs self interactions. So although the production is a rare process in comparison with other Higgs production processes, it has been paid particular attention in last twenty years [98-111].

In the SM the Higgs pair production at the LHC proceeds by the parton process $g g \rightarrow h h$ through the heavy quark induced box diagrams and also through the production of an off-shell Higgs which subsequently splits into two on-shell Higgs bosons (see diagram (1), (2) and (8) of figure 9) [98-100]. The production rate is rather low for $\sqrt{s}=14 \mathrm{TeV}$, about $20 \mathrm{fb}$ at leading order [101] and $35 \mathrm{fb}$ after including the next-to-leading order QCD correction [102-111]. The capability of the LHC to detect this production process was investigated in [112-122] by the channel such as $g g \rightarrow h h \rightarrow b \bar{b} \gamma \gamma, b \bar{b} W W^{*}, b \bar{b} \tau^{+} \tau^{-}$respectively, and it has been shown that the most efficient one is $g g \rightarrow h h \rightarrow b \bar{b} \gamma \gamma$ with 6 signal events over 14 background events expected for $600 \mathrm{fb}^{-1}$ integrated luminosity after considering some elaborate cuts [112-115]. In principle, the capability can be further improved if the recently developed jet substructure technique is applied for the Higgs tagging [123-125].

\footnotetext{
${ }^{2}$ After neglecting gauge interactions, the coupling of $h_{2}$ with dark matter is determined by terms $\lambda \hat{H}_{u}$. $\hat{H}_{d} \hat{S}+\frac{1}{3} \kappa \hat{S}^{3}$ in the superpotential. For a singlet dominated $h_{2}$, the coupling strength is mainly determined by $\lambda$ for bino-like dark matter and by $\kappa$ for singlino-like dark matter. Given the potentially largeness of $\lambda$ and $\kappa$, the strength is moderately large. While for a doublet dominated $h_{2}$, only its coupling with bino-like dark matter is sizable, and it is significantly smaller than the similar coupling for a singlet dominated $h_{2}$ because in contrast with a sizable bino-Higgsino mixing in neutralino mass matrix, there is no bino-singlino mixing.
} 

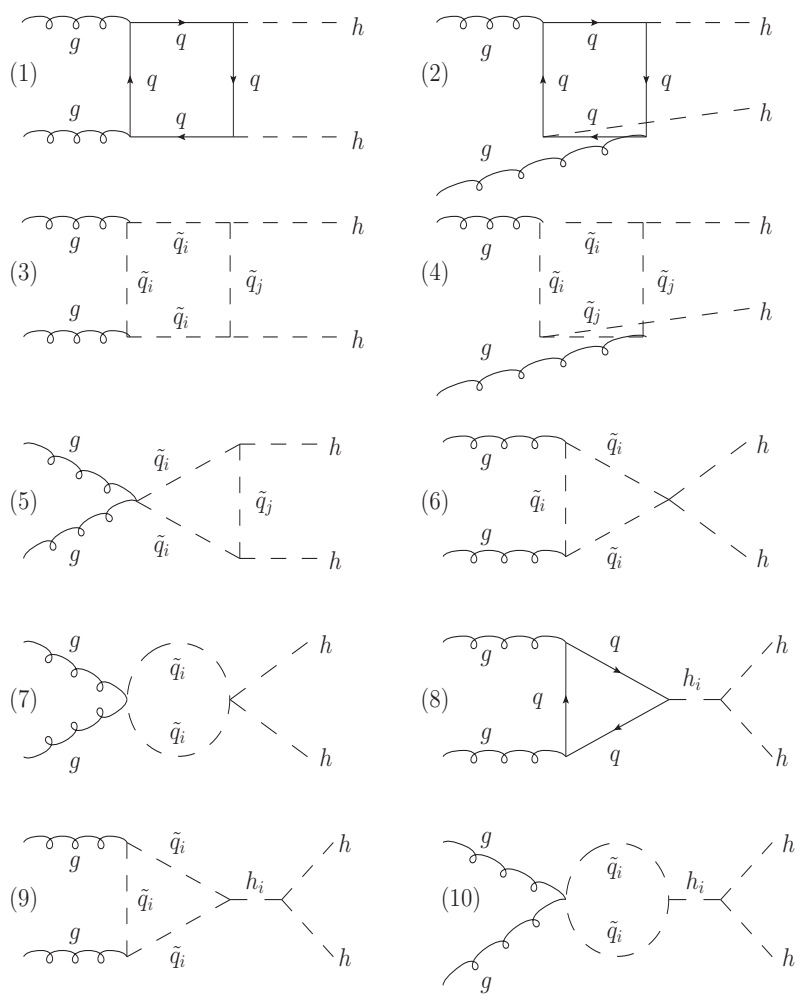

Figure 9. Feynman diagrams for the pair production of the SM-like Higgs boson via gluon fusion in $\lambda$-SUSY with $h_{i}$ denoting a CP-even Higgs $(i=1,2,3)$ and $\tilde{q}_{i, j}(i, j=1,2)$ denoting a squark. The diagrams with initial gluons or final Higgs bosons interchanged are not shown here. For the quarks and squarks we only consider the third generation due to their large Yukawa couplings.

In SUSY the Higgs pair production may also proceed through the diagrams 3-10 in figure 9 with the internal particles in the loops involving the third generation squarks and the intermediating s-channel scalar being any CP-even Higgs boson [126-132]. Since the genuine SUSY contribution to the amplitude is of the same perturbation order as the SM contribution, the SUSY prediction on the production rate may significantly deviate from the SM result. Based on previous studies in this field [58-61, 132, 134], we learn that there are three main mechanisms to enhance the production rate greatly:

- Through the loops mediated by stops [132]. In SUSY, the coupling strength of the $h \tilde{t}_{i}^{*} \tilde{t}_{j}$ interaction is mainly determined by the trilinear soft breaking parameter $A_{t}$, and consequently stops contribute to the pair production in following way [132]

$$
\mathcal{M} \sim \alpha_{s}^{2} Y_{t}^{2}\left(c_{1} \sin ^{2} 2 \theta_{t} \frac{A_{t}^{2}}{m_{\tilde{t}_{1}}^{2}}+c_{2} \frac{A_{t}^{2}}{m_{\tilde{t}_{2}}^{2}}\right),
$$

where $\mathcal{M}$ denotes the amplitude of the stop-induced box diagrams, $Y_{t}$ is top quark Yukawa coupling, $\theta_{t}$ is the mixing angle of stops and $c_{1}, c_{2}$ are dimensionless coefficients determined by detailed loop calculation. It is then obvious that the stop contributions may enhance the pair production rate greatly for a light stop along 


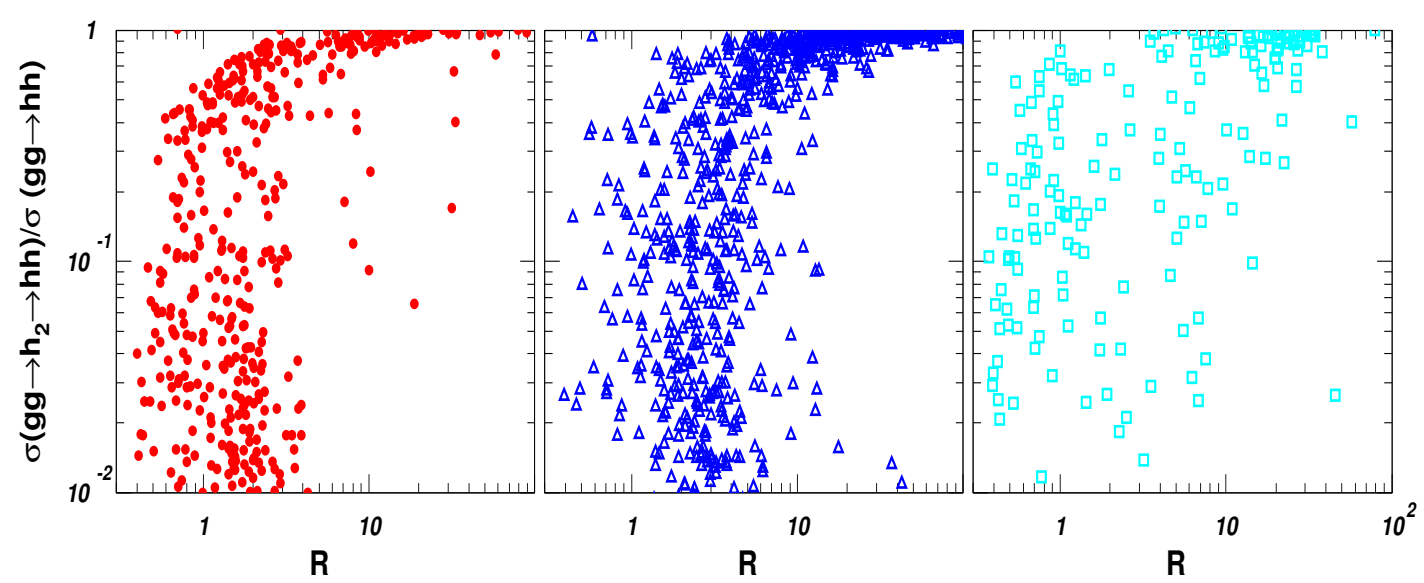

Figure 10. Correlation between the normalized total cross section $\sigma(p p \rightarrow h h)$, $R$, and the pure resonant $s$-channel contribution to the pair production. Results shown in left panel, middle panel and right panel are for Type-I samples, Type-II samples and Type-III samples respectively.

with a large $A_{t}$. Detailed calculation indicates that the corrected cross section may be several times larger than its SM prediction [132].

- Through the resonant effect of a CP-even state $h_{i}$ [58-61]. In SUSY, $h_{i}$ may be onshell produced by $g g$ or $b \bar{b}$ initial state. For $260 \mathrm{GeV} \lesssim m_{h_{i}} \lesssim 400 \mathrm{GeV}$, the production rate is not suppressed by parton distribution function, and meanwhile $h_{i}$ may decay dominantly into $h h$. In this case, the on-shell production of $h_{i}$ can greatly enhance the pair production rate.

In $\lambda$-SUSY, usually only the non-SM doublet dominant $h_{2}$ is pertinent to the enhancement, and its resonance effect on the pair production is estimated by

$$
\sigma(g g \rightarrow h h)(p b) \simeq(12.5 \sim 14.5) \times\left(\cot \beta-\tan \beta A_{\frac{1}{2}}\left(\tau_{b}\right) / A_{\frac{1}{2}}\left(\tau_{t}\right)\right)^{2} \times B r\left(h_{2} \rightarrow h h\right)
$$

where $\tau_{b}=m_{h_{2}}^{2} /\left(4 m_{b}^{2}\right)$ and $\tau_{t}=m_{h_{2}}^{2} /\left(4 m_{t}^{2}\right)$. In getting this estimation, we use the fact that $\sigma\left(g g \rightarrow h_{2}\right)$ at $14-\mathrm{TeV} \mathrm{LHC}$ is about $14.5 \mathrm{pb}(12.5 \mathrm{pb})$ for $m_{h_{2}}=260 \mathrm{GeV}$ $(350 \mathrm{GeV})$ given that the $h_{2}$ has same couplings as the SM Higgs boson to top and bottom quarks [133], and meanwhile neglecting the squark contribution to the $h_{2} g g$ coupling. For $\tan \beta=2$ and $\operatorname{Br}\left(h_{2} \rightarrow h h\right)=60 \%$, one can learn that the rate is about $(1.8 \sim 2.2) \mathrm{pb}$, which is about 100 times larger than the SM prediction.

- Through a large Higgs self coupling [134]. In the SM, the triple self coupling of the Higgs boson plays a minor role in contributing to the pair production due to its relative smallness: $C_{h h h}^{\mathrm{SM}} \simeq 32 \mathrm{GeV}$, and its effect is to cancel the dominant top quark contribution. While if the self coupling is sufficiently enhanced, the situation will change and the self coupling contribution may become dominant. Given that $h$ has same couplings as the SM Higgs boson to top and bottom quarks, and meanwhile neglecting the squark effect to the production, one can roughly estimate the pair 


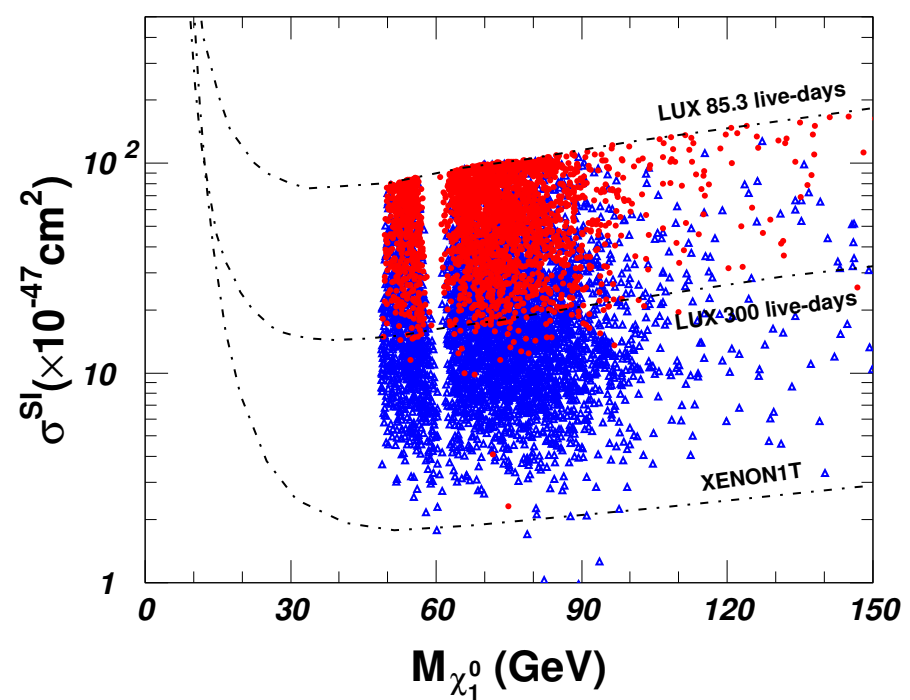

Figure 11. Same as figure 10, but showing the spin-independent $\chi_{1}^{0}$-nucleon scattering cross section as a function of the dark matter only for Type-I and Type-II samples.

production rate by $[134]$

$$
\sigma\left(g g \rightarrow h^{*} \rightarrow h h\right)(p b) \simeq\left(C_{h h h} / S M-2.5\right)^{2} / 1.5^{2} \times 0.019,
$$

where $C_{h h h} / S M$ is the normalized self coupling of the SM-like Higgs boson in $\lambda$ SUSY. This estimation coincides in magnitude with our precise results presented in table 2 for the benchmark points $\mathrm{P} 1$ and $\mathrm{P} 2$.

In the following, we define the normalized Higgs pair production rate by $R=\sigma(p p \rightarrow$ $h h) /\left(\left.\sigma_{\mathrm{SM}}^{\mathrm{LO}}(p p \rightarrow h h)\right|_{m_{h}=125 \mathrm{GeV}}\right) \simeq \sigma(p p \rightarrow h h) /(19 \mathrm{fb})$ for the convenience to present our results, and use the same code as [132] to calculate the cross section of $p p \rightarrow h h$ in $\lambda$-SUSY. In figure 10, we present the value of $R$ for Type-I, Type-II and Type-III samples in leftpanel, middle panel and right panel respectively. In order to emphasize the resonance $h_{2}$ contribution, we also calculate the process $g g \rightarrow h_{2} \rightarrow h h$ separately, and present the ratio $\sigma\left(p p \rightarrow h_{2} \rightarrow h h\right) / \sigma(p p \rightarrow h h)$ in the same figure. This figure indicates that the Higgs pair production rate may get enhanced by more ten times either through the resonance $h_{2}$ effect (corresponding to points with the ratio around one in the figure) or through the large self coupling contribution (corresponding to points with the ratio significantly below one). Especially, in some extreme cases we find that the pair production may get enhanced by more than 100 times for the Type-II sample, which seems impossible in the MSSM [132].

Before we end our discussion, we'd like to point that the spin-independent cross section for dark matter scattering off nucleon may be moderately large since the couplings of the CP-even states $h_{i}$ with dark matter can be enhanced by a large $\lambda$ [38-40]. In figure 11, we show such a rate as a function of the dark matter mass. In calculating the cross section, we use the formula presented in [135] by choosing a rather low $f_{T s}, f_{T s}=0.025$, which represents the strange quark component in nucleon. This figure indicates that given LUX experiment with 300 live-days data, most of the Type-I samples will be excluded in case 
that no dark matter signal is observed. Furthermore, if the updated XENON1T does not detect any signal of the dark matter, nearly all samples of $\lambda$-SUSY will be excluded. These facts tell us that the dark matter direct experiments in parallel with collider experiment such as the LHC can serve as a powerful tools in testing the framework of $\lambda$-SUSY.

\section{Summary and conclusions}

Since the first hint of the $125 \mathrm{GeV}$ Higgs-like particle appeared at the end of 2011, the unnaturalness of the MSSM in predicting the Higgs mass and also the absence of SUSY signal at the LHC have motivated more and more interests of the non-minimal realizations of SUSY. This revived the $\lambda$-SUSY theory, which corresponds to the NMSSM with a large $\lambda$ around one. In the framework of the $\lambda$-SUSY, the Higgs mass can be around $125 \mathrm{GeV}$ even without the large top-squark radiative correction, and meanwhile the sensitivity of the weak scale to stop masses is reduced by a factor of $(g / \lambda)^{2}$ in comparison with the MSSM, which means that the lower bound on the stop mass imposed by the LHC direct searches has a weaker implication on fine-tuning in this model than in the MSSM or the NMSSM with a low $\lambda$. Due to these advantages, the $\lambda$-SUSY has been considered as a most natural realization of SUSY [41-47].

In order to implement the constraints on the $\lambda$-SUSY in a better way, we consider the Higgs data recently updated by the ATLAS and CMS collaborations, for which the consistency of the two group results has been improved greatly. We also define two quantities to measure the naturalness of the parameter points. After these preparations, we scan the parameter space of the $\lambda$-SUSY by considering various constraints, then investigate the features of its Higgs sector in physical parameter region. As is shown in this work, the improvement of the two constraints is really necessary. For example, we find the values of the Higgs $\chi^{2}$ obtained with the latest Higgs data are significantly reduced than before, and the naturalness argument does play an important role in selecting the parameter space of the $\lambda$-SUSY.

For the SM-like Higgs boson $h$, we have following conclusions:

- Current Higgs data still allow for a sizable singlet component in $h$, which at most reaches $25 \%$, while the non-SM doublet component is forbidden to be larger than $1 \%$.

- Due the latest Higgs data, the normalized couplings such as $C_{h \gamma \gamma} / S M, C_{h Z Z} / S M$ and $C_{h \bar{t} t} / S M$ are limited within $15 \%$ deviation from unity at $95 \%$ C.L.. Compared with the similar fit results in 2012, the optimal values of the couplings in the new fit are shifted significantly.

- Interestingly, the strength of the triple self coupling of $h$ may get enhanced by a factor over 10, and naturalness can limit such a possibility.

For the next-to-lightest CP-even Higgs boson $h_{2}$, we find

- For $m_{h_{2}} \leq 500 \mathrm{GeV}, h_{2}$ in most cases is either highly non-SM doublet dominated or highly singlet dominated. This feature enables us to express the couplings of $h_{2}$ in a simple analytic way. 
- For the non-SM double dominated $h_{2}$, it may be as light as $200 \mathrm{GeV}$, which seems impossible in the MSSM. As for its coupling, we find $\left|C_{h \bar{t}} / S M\right| \geq 0.2$, and in optimal case the normalized coupling is just slightly below 1 . On the other hand, $\left|C_{h \bar{b} b} / S M\right|$ is usually larger than one with its maximum value reaching 6 . As a result, the $h_{2} g g$ coupling may be comparable with the SM hgg coupling for a low $\tan \beta$.

- For the singlet dominated $h_{2}$, although it may be as light as $150 \mathrm{GeV}$, its couplings with SM fermions is usually rather weak, so is of less interest in phenomenology study.

- For either the doublet dominated $h_{2}$ or the singlet dominated $h_{2}$, the strength of the $h_{2} h h$ interaction may be quite large. Consequently, $h_{2} \rightarrow h h$ can act as the dominant decay channel of $h_{2}$.

- Naturalness disfavors a $h_{2}$ with mass at several TeV regardless its field components.

We also investigate the $h$ pair production process, and we show three mechanisms to enhance the rate greatly, i.e. by stop-induced box diagrams, by s-channel resonant $h_{2}$ effect and by large self coupling of $h$. With these mechanisms, we conclude that the $h$ pair production rate in $\lambda$-SUSY may be enhanced by more than 100 times compared with its SM prediction.

In summary, in this work we obtained two possible characteristic features of $\lambda$-SUSY in the experimentally allowed parameter space: 1) the triple self coupling of the SM-like Higgs boson may get enhanced by a factor over 10 in comparison with its SM prediction; 2) the pair production of the SM-like Higgs boson at the LHC may be two orders larger than its SM prediction. These two features seems to be unachievable in the MSSM and in the NMSSM with a low $\lambda$, and should be tested at the future LHC.

\section{Acknowledgments}

We thank Ben O'Leary, Florian Staub, Jinmin Yang, C.-P. Yuan, Haijing Zhou and Jingya Zhu for helpful discussions. This work was supported by the National Natural Science Foundation of China (NNSFC) under grant No. 11222548 and 11275245.

Open Access. This article is distributed under the terms of the Creative Commons Attribution License (CC-BY 4.0), which permits any use, distribution and reproduction in any medium, provided the original author(s) and source are credited.

\section{References}

[1] ATLAS collaboration, Observation of a new particle in the search for the standard model Higgs boson with the ATLAS detector at the LHC, Phys. Lett. B 716 (2012) 1 [arXiv: 1207.7214] [INSPIRE].

[2] CMS collaboration, Observation of a new boson at a mass of $125 \mathrm{GeV}$ with the CMS experiment at the LHC, Phys. Lett. B $\mathbf{7 1 6}$ (2012) 30 [arXiv:1207.7235] [INSPIRE]. 
[3] ATLAS collaboration, Combined coupling measurements of the Higgs-like boson with the ATLAS detector using up to $25 \mathrm{fb}^{-1}$ of proton-proton collision data, ATLAS-CONF-2013-034 (2013).

[4] CMS collaboration, Combination of standard model Higgs boson searches and measurements of the properties of the new boson with a mass near 125 GeV, CMS-PAS-HIG-13-005 (2013).

[5] ATLAS collaboration, Measurement of Higgs boson production in the diphoton decay channel in pp collisions at center-of-mass energies of 7 and 8 TeV with the ATLAS detector, arXiv: 1408.7084 [INSPIRE].

[6] ATLAS collaboration, Measurements of Higgs boson production and couplings in the four-lepton channel in pp collisions at center-of-mass energies of 7 and 8 TeV with the ATLAS detector, arXiv:1408.5191 [INSPIRE].

[7] ATLAS collaboration, ATLAS interpretation of the combined measurements of coupling properties of the Higgs boson in terms of its production cross sections, ATL-PHYS-PUB-2014-009 (2014).

[8] CMS collaboration, Precise determination of the mass of the Higgs boson and studies of the compatibility of its couplings with the standard model, CMS-PAS-HIG-14-009 (2014).

[9] H. Baer, V. Barger and A. Mustafayev, Implications of a 125 GeV Higgs scalar for LHC SUSY and neutralino dark matter searches, Phys. Rev. D 85 (2012) 075010 [arXiv:1112.3017] [INSPIRE].

[10] S. Heinemeyer, O. Stal and G. Weiglein, Interpreting the LHC Higgs search results in the MSSM, Phys. Lett. B 710 (2012) 201 [arXiv:1112.3026] [INSPIRE].

[11] A. Arbey, M. Battaglia, A. Djouadi, F. Mahmoudi and J. Quevillon, Implications of a 125 GeV Higgs for supersymmetric models, Phys. Lett. B 708 (2012) 162 [arXiv:1112.3028] [INSPIRE].

[12] P. Draper, P. Meade, M. Reece and D. Shih, Implications of a 125 GeV Higgs for the MSSM and low-scale SUSY breaking, Phys. Rev. D 85 (2012) 095007 [arXiv:1112.3068] [INSPIRE].

[13] M. Carena, S. Gori, N.R. Shah and C.E.M. Wagner, A 125 GeV SM-like Higgs in the MSSM and the $\gamma \gamma$ rate, JHEP 03 (2012) 014 [arXiv: 1112.3336] [INSPIRE].

[14] S. Akula, B. Altunkaynak, D. Feldman, P. Nath and G. Peim, Higgs boson mass predictions in SUGRA unification, recent $L H C-7$ results and dark matter, Phys. Rev. D 85 (2012) 075001 [arXiv:1112.3645] [InSPIRE].

[15] M. Kadastik, K. Kannike, A. Racioppi and M. Raidal, Implications of the 125 GeV Higgs boson for scalar dark matter and for the CMSSM phenomenology, JHEP 05 (2012) 061 [arXiv:1112.3647] [INSPIRE].

[16] J. Cao, Z. Heng, D. Li and J.M. Yang, Current experimental constraints on the lightest Higgs boson mass in the constrained MSSM, Phys. Lett. B 710 (2012) 665 [arXiv:1112.4391] [INSPIRE].

[17] Z. Kang, T. Li, T. Liu, C. Tong and J.M. Yang, A heavy SM-like Higgs and a light stop from Yukawa-deflected gauge mediation, Phys. Rev. D 86 (2012) 095020 [arXiv:1203.2336] [INSPIRE].

[18] N.D. Christensen, T. Han and S. Su, MSSM Higgs bosons at The LHC, Phys. Rev. D 85 (2012) 115018 [arXiv:1203.3207] [INSPIRE].

[19] H. An, T. Liu and L.-T. Wang, 125 GeV Higgs boson, enhanced di-photon rate and gauged $\mathrm{U}(1)_{P Q}$-extended MSSM, Phys. Rev. D 86 (2012) 075030 [arXiv:1207.2473] [INSPIRE]. 
[20] C. Boehm, P.S.B. Dev, A. Mazumdar and E. Pukartas, Naturalness of light neutralino dark matter in pMSSM after LHC, XENON100 and Planck data, JHEP 06 (2013) 113 [arXiv: 1303.5386] [INSPIRE].

[21] U. Ellwanger, A Higgs boson near $125 \mathrm{GeV}$ with enhanced di-photon signal in the NMSSM, JHEP 03 (2012) 044 [arXiv:1112.3548] [INSPIRE].

[22] J.F. Gunion, Y. Jiang and S. Kraml, The constrained NMSSM and Higgs near $125 \mathrm{GeV}$, Phys. Lett. B 710 (2012) 454 [arXiv:1201.0982] [INSPIRE].

[23] S.F. King, M. Muhlleitner and R. Nevzorov, NMSSM Higgs benchmarks near $125 \mathrm{GeV}$, Nucl. Phys. B 860 (2012) 207 [arXiv:1201.2671] [INSPIRE].

[24] J.-J. Cao, Z.-X. Heng, J.M. Yang, Y.-M. Zhang and J.-Y. Zhu, A SM-like Higgs near $125 \mathrm{GeV}$ in low energy SUSY: a comparative study for MSSM and NMSSM, JHEP 03 (2012) 086 [arXiv: 1202.5821] [INSPIRE].

[25] D.A. Vasquez et al., The $125 \mathrm{GeV}$ Higgs in the NMSSM in light of LHC results and astrophysics constraints, Phys. Rev. D 86 (2012) 035023 [arXiv:1203.3446] [INSPIRE].

[26] K. Schmidt-Hoberg and F. Staub, Enhanced $h \rightarrow \gamma \gamma$ rate in MSSM singlet extensions, JHEP 10 (2012) 195 [arXiv:1208.1683] [INSPIRE].

[27] R. Benbrik et al., Confronting the MSSM and the NMSSM with the discovery of a signal in the two photon channel at the LHC, Eur. Phys. J. C 72 (2012) 2171 [arXiv:1207.1096] [INSPIRE].

[28] G. Bélanger et al., Higgs bosons at 98 and $125 \mathrm{GeV}$ at LEP and the LHC, JHEP 01 (2013) 069 [arXiv:1210.1976] [INSPIRE].

[29] K. Choi, S.H. Im, K.S. Jeong and M. Yamaguchi, Higgs mixing and diphoton rate enhancement in NMSSM models, JHEP 02 (2013) 090 [arXiv:1211.0875] [INSPIRE].

[30] S.F. King, M. Mühlleitner, R. Nevzorov and K. Walz, Natural NMSSM Higgs bosons, Nucl. Phys. B 870 (2013) 323 [arXiv:1211.5074] [INSPIRE].

[31] N.D. Christensen, T. Han, Z. Liu and S. Su, Low-mass Higgs bosons in the NMSSM and their LHC implications, JHEP 08 (2013) 019 [arXiv:1303.2113] [INSPIRE].

[32] M. Badziak, M. Olechowski and S. Pokorski, New regions in the NMSSM with a $125 \mathrm{GeV}$ Higgs, JHEP 06 (2013) 043 [arXiv: 1304.5437] [INSPIRE].

[33] S. Moretti, S. Munir and P. Poulose, $125 \mathrm{GeV}$ Higgs boson signal within the complex NMSSM, Phys. Rev. D 89 (2014) 015022 [arXiv: 1305.0166] [INSPIRE].

[34] W. Wang, J.M. Yang and L.L. You, Higgs boson mass in NMSSM with right-handed neutrino, JHEP 07 (2013) 158 [arXiv:1303.6465] [INSPIRE].

[35] J. Cao, Z. Heng, J.M. Yang and J. Zhu, Status of low energy SUSY models confronted with the LHC $125 \mathrm{GeV}$ Higgs data, JHEP 10 (2012) 079 [arXiv:1207.3698] [INSPIRE].

[36] P. Bechtle et al., MSSM interpretations of the LHC discovery: light or heavy Higgs?, Eur. Phys. J. C 73 (2013) 2354 [arXiv:1211.1955] [INSPIRE].

[37] Z. Kang, J. Li and T. Li, On naturalness of the MSSM and NMSSM, JHEP 11 (2012) 024 [arXiv:1201.5305] [INSPIRE].

[38] U. Ellwanger, C. Hugonie and A.M. Teixeira, The next-to-minimal supersymmetric standard model, Phys. Rept. 496 (2010) 1 [arXiv:0910.1785] [INSPIRE].

[39] M. Maniatis, The next-to-minimal supersymmetric extension of the standard model reviewed, Int. J. Mod. Phys. A 25 (2010) 3505 [arXiv:0906.0777] [INSPIRE]. 
[40] S.F. King and P.L. White, Resolving the constrained minimal and next-to-minimal supersymmetric standard models, Phys. Rev. D 52 (1995) 4183 [hep-ph/9505326] [INSPIRE].

[41] L.J. Hall, D. Pinner and J.T. Ruderman, A natural SUSY Higgs near $126 \mathrm{GeV}$, JHEP 04 (2012) 131 [arXiv:1112.2703] [INSPIRE].

[42] M. Perelstein and B. Shakya, XENON100 implications for naturalness in the MSSM, NMSSM and $\lambda$-supersymmetry model, Phys. Rev. D 88 (2013) 075003 [arXiv:1208.0833] [INSPIRE].

[43] K. Agashe, Y. Cui and R. Franceschini, Natural islands for a $125 \mathrm{GeV}$ Higgs in the scale-invariant NMSSM, JHEP 02 (2013) 031 [arXiv: 1209.2115] [INSPIRE].

[44] T. Gherghetta, B. von Harling, A.D. Medina and M.A. Schmidt, The scale-invariant NMSSM and the $126 \mathrm{GeV}$ Higgs boson, JHEP 02 (2013) 032 [arXiv:1212.5243] [INSPIRE].

[45] M.Y. Binjonaid and S.F. King, Naturalness of scale-invariant NMSSMs with and without extra matter, Phys. Rev. D 90 (2014) 055020 [arXiv: 1403.2088] [INSPIRE].

[46] R. Barbieri, D. Buttazzo, K. Kannike, F. Sala and A. Tesi, One or more Higgs bosons?, Phys. Rev. D 88 (2013) 055011 [arXiv:1307.4937] [INSPIRE].

[47] R. Barbieri, D. Buttazzo, K. Kannike, F. Sala and A. Tesi, Exploring the Higgs sector of a most natural NMSSM, Phys. Rev. D 87 (2013) 115018 [arXiv:1304.3670] [INSPIRE].

[48] M. Farina, M. Perelstein and B. Shakya, Higgs couplings and naturalness in $\lambda$-SUSY, JHEP 04 (2014) 108 [arXiv:1310.0459] [INSPIRE].

[49] R. Harnik, G.D. Kribs, D.T. Larson and H. Murayama, The minimal supersymmetric fat Higgs model, Phys. Rev. D 70 (2004) 015002 [hep-ph/0311349] [INSPIRE].

[50] S. Chang, C. Kilic and R. Mahbubani, The new fat Higgs: slimmer and more attractive, Phys. Rev. D 71 (2005) 015003 [hep-ph/0405267] [INSPIRE].

[51] R. Barbieri, L.J. Hall, A.Y. Papaioannou, D. Pappadopulo and V.S. Rychkov, An alternative NMSSM phenomenology with manifest perturbative unification, JHEP 03 (2008) 005 [arXiv:0712.2903] [InSPIRE].

[52] E. Hardy, J. March-Russell and J. Unwin, Precision unification in $\lambda$ SUSY with a $125 \mathrm{GeV}$ Higgs, JHEP 10 (2012) 072 [arXiv:1207.1435] [INSPIRE].

[53] R. Barbieri, L.J. Hall, Y. Nomura and V.S. Rychkov, Supersymmetry without a light Higgs boson, Phys. Rev. D 75 (2007) 035007 [hep-ph/0607332] [INSPIRE].

[54] J. Cao and J.M. Yang, Current experimental constraints on NMSSM with large $\lambda$, Phys. Rev. D 78 (2008) 115001 [arXiv:0810.0989] [INSPIRE].

[55] A. Djouadi and J. Quevillon, The MSSM Higgs sector at a high $M_{S U S Y}$ : reopening the low $\tan \beta$ regime and heavy Higgs searches, JHEP 10 (2013) 028 [arXiv: 1304.1787] [INSPIRE].

[56] ATLAS collaboration, Search for neutral Higgs bosons of the minimal supersymmetric standard model in pp collisions at $\sqrt{s}=8 \mathrm{TeV}$ with the ATLAS detector, ATLAS-CONF-2014-049 (2014).

[57] CMS collaboration, Higgs to tau tau (MSSM), CMS-PAS-HIG-13-021 (2013).

[58] J. Liu, X.-P. Wang and S.-h. Zhu, Discovering extra Higgs boson via pair production of the SM-like Higgs bosons, arXiv:1310.3634 [INSPIRE].

[59] J.M. No and M. Ramsey-Musolf, Probing the Higgs portal at the LHC through resonant di-Higgs production, Phys. Rev. D 89 (2014) 095031 [arXiv:1310.6035] [INSPIRE]. 
[60] B. Bhattacherjee and A. Choudhury, The role of MSSM heavy Higgs production in the self coupling measurement of $125 \mathrm{GeV}$ Higgs boson at the LHC, arXiv:1407.6866 [INSPIRE].

[61] V. Barger, L.L. Everett, C.B. Jackson, A. Peterson and G. Shaughnessy, New physics in resonant production of Higgs boson pairs, arXiv:1408.0003 [INSPIRE].

[62] U. Ellwanger, G. Espitalier-Noel and C. Hugonie, Naturalness and fine tuning in the NMSSM: implications of early LHC results, JHEP 09 (2011) 105 [arXiv:1107.2472] [INSPIRE].

[63] U. Ellwanger and C. Hugonie, NMHDECAY 2.0: an updated program for sparticle masses, Higgs masses, couplings and decay widths in the NMSSM,

Comput. Phys. Commun. 175 (2006) 290 [hep-ph/0508022] [INSPIRE].

[64] U. Ellwanger, J.F. Gunion and C. Hugonie, NMHDECAY: a Fortran code for the Higgs masses, couplings and decay widths in the NMSSM, JHEP 02 (2005) 066 [hep-ph/0406215] [INSPIRE].

[65] ATLAS collaboration, Search for direct stop production in events with missing transverse momentum and two b-jets using $12.8 \mathrm{fb}^{-1}$ of pp collisions at $\sqrt{\mathrm{s}}=8 \mathrm{TeV}$ with the ATLAS detector, ATLAS-CONF-2013-001 (2013).

[66] CMS collaboration, Search for direct top squark pair production in events with a single isolated lepton, jets and missing transverse energy at $\sqrt{(s)}=8 \mathrm{TeV}$, CMS-PAS-SUS-12-023 (2012).

[67] J. Cao, C. Han, L. Wu, J.M. Yang and Y. Zhang, Probing natural SUSY from stop pair production at the LHC, JHEP 11 (2012) 039 [arXiv:1206.3865] [INSPIRE].

[68] X.-J. Bi, Q.-S. Yan and P.-F. Yin, Light stop/sbottom pair production searches in the NMSSM, Phys. Rev. D 87 (2013) 035007 [arXiv:1209.2703] [INSPIRE].

[69] C. Han, K.-i. Hikasa, L. Wu, J.M. Yang and Y. Zhang, Current experimental bounds on stop mass in natural SUSY, JHEP 10 (2013) 216 [arXiv:1308.5307] [INSPIRE].

[70] M.D. Goodsell, K. Nickel and F. Staub, On the two-loop corrections to the Higgs masses in the NMSSM, arXiv:1411.4665 [INSPIRE].

[71] P. Bechtle, O. Brein, S. Heinemeyer, G. Weiglein and K.E. Williams, HiggsBounds: confronting arbitrary Higgs sectors with exclusion bounds from LEP and the Tevatron, Comput. Phys. Commun. 181 (2010) 138 [arXiv:0811.4169] [INSPIRE].

[72] P. Bechtle, O. Brein, S. Heinemeyer, G. Weiglein and K.E. Williams, HiggsBounds 2.0.0: confronting neutral and charged Higgs sector predictions with exclusion bounds from LEP and the Tevatron, Comput. Phys. Commun. 182 (2011) 2605 [arXiv:1102.1898] [INSPIRE].

[73] J.E. Camargo-Molina, B. O'Leary, W. Porod and F. Staub, Vevacious: a tool for finding the global minima of one-loop effective potentials with many scalars, Eur. Phys. J. C 73 (2013) 2588 [arXiv:1307.1477] [inSPIRE].

[74] D. Chowdhury, R.M. Godbole, K.A. Mohan and S.K. Vempati, Charge and color breaking constraints in MSSM after the Higgs discovery at LHC, JHEP 02 (2014) 110 [arXiv:1310.1932] [INSPIRE].

[75] N. Blinov and D.E. Morrissey, Vacuum stability and the MSSM Higgs mass, JHEP 03 (2014) 106 [arXiv: 1310.4174] [INSPIRE].

[76] M. Bobrowski, G. Chalons, W.G. Hollik and U. Nierste, Vacuum stability of the effective Higgs potential in the minimal supersymmetric standard model, Phys. Rev. D 90 (2014) 035025 [arXiv:1407.2814] [INSPIRE]. 
[77] U. Chattopadhyay and A. Dey, Exploring MSSM for charge and color breaking and other constraints in the context of Higgs@125 GeV, arXiv:1409.0611 [INSPIRE].

[78] F. Staub, SARAH, arXiv:0806.0538 [INSPIRE].

[79] F. Staub, Automatic calculation of supersymmetric renormalization group equations and self energies, Comput. Phys. Commun. 182 (2011) 808 [arXiv:1002.0840] [INSPIRE].

[80] F. Staub, SARAH 3.2: Dirac Gauginos, UFO output and more, Comp. Phys. Commun. 184 (2013) 1792 [arXiv:1207.0906] [INSPIRE].

[81] F. Staub, SARAH 4: a tool for (not only SUSY) model builders, Comput. Phys. Commun. 185 (2014) 1773 [arXiv:1309.7223] [InSPIRE].

[82] W. Porod, SPheno, a program for calculating supersymmetric spectra, SUSY particle decays and SUSY particle production at $e^{+} e^{-}$colliders, Comput. Phys. Commun. 153 (2003) 275 [hep-ph/0301101] [INSPIRE].

[83] W. Porod and F. Staub, SPheno 3.1: extensions including flavour, CP-phases and models beyond the MSSM, Comput. Phys. Commun. 183 (2012) 2458 [arXiv:1104.1573] [INSPIRE].

[84] CDF Collaboration, D0 collaboration, T. Aaltonen et al., Higgs boson studies at the Tevatron, Phys. Rev. D 88 (2013) 052014 [arXiv: 1303.6346] [INSPIRE].

[85] ATLAS collaboration, Measurement of the Higgs boson mass from the $H \rightarrow \gamma \gamma$ and $H \rightarrow Z Z^{*} \rightarrow 4 \ell$ channels with the ATLAS detector using $25 \mathrm{fb}^{-1}$ of pp collision data, Phys. Rev. D 90 (2014) 052004 [arXiv:1406.3827] [InSPIRE].

[86] J.R. Espinosa, C. Grojean, M. Muhlleitner and M. Trott, Fingerprinting Higgs suspects at the LHC, JHEP 05 (2012) 097 [arXiv: 1202.3697] [INSPIRE].

[87] P.P. Giardino, K. Kannike, M. Raidal and A. Strumia, Reconstructing Higgs boson properties from the LHC and Tevatron data, JHEP 06 (2012) 117 [arXiv:1203.4254] [INSPIRE].

[88] K. Cheung, J.S. Lee and P.-Y. Tseng, Higgs precision analysis updates 2014, Phys. Rev. D 90 (2014) 095009 [arXiv:1407.8236] [INSPIRE].

[89] F. Boudjema et al., On the presentation of the LHC Higgs results, arXiv:1307.5865 [INSPIRE].

[90] K. Cheung, J.S. Lee and P.-Y. Tseng, Higgs precision (Higgcision) era begins, JHEP 05 (2013) 134 [arXiv: 1302.3794] [INSPIRE].

[91] G. Bélanger, B. Dumont, U. Ellwanger, J.F. Gunion and S. Kraml, Higgs couplings at the end of 2012, JHEP 02 (2013) 053 [arXiv:1212.5244] [INSPIRE].

[92] J. Cao, F. Ding, C. Han, J.M. Yang and J. Zhu, A light Higgs scalar in the NMSSM confronted with the latest LHC Higgs data, JHEP 11 (2013) 018 [arXiv:1309.4939] [INSPIRE].

[93] P. Bechtle, S. Heinemeyer, O. Stål, T. Stefaniak and G. Weiglein, HiggsSignals: confronting arbitrary Higgs sectors with measurements at the Tevatron and the LHC,

Eur. Phys. J. C 74 (2014) 2711 [arXiv:1305.1933] [INSPIRE].

[94] O. Stål and T. Stefaniak, Constraining extended Higgs sectors with HiggsSignals, PoS (EPS-HEP 2013) 314 [arXiv: 1310.4039] [INSPIRE].

[95] A. Djouadi, The anatomy of electro-weak symmetry breaking. II. The Higgs bosons in the minimal supersymmetric model, Phys. Rept. 459 (2008) 1 [hep-ph/0503173] [INSPIRE]. 
[96] A. Arbey, M. Battaglia and F. Mahmoudi, Supersymmetric heavy Higgs bosons at the LHC, Phys. Rev. D 88 (2013) 015007 [arXiv: 1303.7450] [inSPIRE].

[97] K.J. Bae, H. Baer, V. Barger, D. Mickelson and M. Savoy, Implications of naturalness for the heavy Higgs bosons of supersymmetry, Phys. Rev. D 90 (2014) 075010 [arXiv: 1407.3853] [INSPIRE].

[98] E.W.N. Glover and J.J. van der Bij, Higgs boson pair production via gluon fusion, Nucl. Phys. B 309 (1988) 282 [inSPIRE].

[99] D.A. Dicus, C. Kao and S.S.D. Willenbrock, Higgs boson pair production from gluon fusion, Phys. Lett. B 203 (1988) 457 [INSPIRE].

[100] T. Plehn, M. Spira and P.M. Zerwas, Pair production of neutral Higgs particles in gluon-gluon collisions, Nucl. Phys. B 479 (1996) 46 [Erratum ibid. B 531 (1998) 655] [hep-ph/9603205] [INSPIRE].

[101] A. Djouadi, W. Kilian, M. Muhlleitner and P.M. Zerwas, Production of neutral Higgs boson pairs at LHC, Eur. Phys. J. C 10 (1999) 45 [hep-ph/9904287] [InSPIRE].

[102] S. Dawson, S. Dittmaier and M. Spira, Neutral Higgs boson pair production at hadron colliders: QCD corrections, Phys. Rev. D 58 (1998) 115012 [hep-ph/9805244] [INSPIRE].

[103] J. Baglio et al., The measurement of the Higgs self-coupling at the LHC: theoretical status, JHEP 04 (2013) 151 [arXiv: 1212.5581] [INSPIRE].

[104] D.Y. Shao, C.S. Li, H.T. Li and J. Wang, Threshold resummation effects in Higgs boson pair production at the LHC, JHEP 07 (2013) 169 [arXiv:1301.1245] [INSPIRE].

[105] D. de Florian and J. Mazzitelli, Two-loop virtual corrections to Higgs pair production, Phys. Lett. B 724 (2013) 306 [arXiv:1305.5206] [INSPIRE].

[106] J. Grigo, J. Hoff, K. Melnikov and M. Steinhauser, On the Higgs boson pair production at the LHC, Nucl. Phys. B 875 (2013) 1 [arXiv:1305.7340] [InSPIRE].

[107] D. de Florian and J. Mazzitelli, Higgs boson pair production at next-to-next-to-leading order in QCD, Phys. Rev. Lett. 111 (2013) 201801 [arXiv: 1309.6594] [INSPIRE].

[108] R. Frederix et al., Higgs pair production at the LHC with NLO and parton-shower effects, Phys. Lett. B 732 (2014) 142 [arXiv:1401.7340] [INSPIRE].

[109] L. Liu-Sheng et al., NNLO QCD corrections to Higgs pair production via vector boson fusion at hadron colliders, Phys. Rev. D 89 (2014) 073001 [arXiv:1401.7754] [INSPIRE].

[110] J. Grigo, K. Melnikov and M. Steinhauser, Virtual corrections to Higgs boson pair production in the large top quark mass limit, Nucl. Phys. B $\mathbf{8 8 8}$ (2014) 17 [arXiv: 1408.2422] [INSPIRE].

[111] S.F. King, M. Mühlleitner, R. Nevzorov and K. Walz, Discovery prospects for NMSSM Higgs bosons at the high-energy Large Hadron Collider, Phys. Rev. D 90 (2014) 095014 [arXiv: 1408.1120] [INSPIRE].

[112] U. Baur, T. Plehn and D.L. Rainwater, Probing the Higgs selfcoupling at hadron colliders using rare decays, Phys. Rev. D 69 (2004) 053004 [hep-ph/0310056] [INSPIRE].

[113] W. Yao, Studies of measuring Higgs self-coupling with $H H \rightarrow b \bar{b} \gamma \gamma$ at the future hadron colliders, arXiv:1308.6302 [INSPIRE].

[114] V. Barger, L.L. Everett, C.B. Jackson and G. Shaughnessy, Higgs-pair production and measurement of the triscalar coupling at LHC(8,14), Phys. Lett. B 728 (2014) 433 [arXiv: 1311.2931] [INSPIRE]. 
[115] ATLAS collaboration, Search for Higgs boson pair production in the $\gamma \gamma b \bar{b}$ final state using pp collision data at $\sqrt{s}=8 \mathrm{TeV}$ from the ATLAS detector, arXiv:1406.5053 [INSPIRE].

[116] A. Papaefstathiou, L.L. Yang and J. Zurita, Higgs boson pair production at the LHC in the $b \bar{b} W^{+} W^{-}$channel, Phys. Rev. D 87 (2013) 011301 [arXiv: 1209.1489] [InSPIRE].

[117] F. Goertz, A. Papaefstathiou, L.L. Yang and J. Zurita, Higgs boson self-coupling measurements using ratios of cross sections, JHEP 06 (2013) 016 [arXiv:1301.3492] [INSPIRE].

[118] M.J. Dolan, C. Englert and M. Spannowsky, Higgs self-coupling measurements at the LHC, JHEP 10 (2012) 112 [arXiv:1206.5001] [INSPIRE].

[119] N.D. Christensen, T. Han and T. Li, Pair production of MSSM Higgs bosons in the non-decoupling region at the LHC, Phys. Rev. D 86 (2012) 074003 [arXiv:1206.5816] [INSPIRE].

[120] R. Contino et al., Anomalous couplings in double Higgs production, JHEP 08 (2012) 154 [arXiv:1205.5444] [INSPIRE].

[121] Q. Li, Q.-S. Yan and X. Zhao, Higgs pair production: improved description by matrix element matching, Phys. Rev. D 89 (2014) 033015 [arXiv: 1312.3830] [InSPIRE].

[122] D.E. Ferreira de Lima, A. Papaefstathiou and M. Spannowsky, Standard model Higgs boson pair production in the $(b \bar{b})(b \bar{b})$ final state, JHEP 08 (2014) 030 [arXiv:1404.7139] [INSPIRE].

[123] J.M. Butterworth, A.R. Davison, M. Rubin and G.P. Salam, Jet substructure as a new Higgs search channel at the LHC, Phys. Rev. Lett. 100 (2008) 242001 [arXiv:0802.2470] [INSPIRE].

[124] M. Gouzevitch et al., Scale-invariant resonance tagging in multijet events and new physics in Higgs pair production, JHEP 07 (2013) 148 [arXiv:1303.6636] [INSPIRE].

[125] A.J. Barr, M.J. Dolan, C. Englert and M. Spannowsky, Di-Higgs final states augMT2ed Selecting hh events at the high luminosity LHC, Phys. Lett. B 728 (2014) 308 [arXiv:1309.6318] [INSPIRE].

[126] S.-H. Zhu, C.-S. Li and C.-S. Gao, Lightest neutral Higgs pair production in photon-photon collisions in the minimal supersymmetric standard model, Phys. Rev. D 58 (1998) 015006 [hep-ph/9710424] [INSPIRE].

[127] A. Belyaev, M. Drees and J.K. Mizukoshi, Supersymmetric Higgs boson pair production: discovery prospects at hadron colliders, Eur. Phys. J. C 17 (2000) 337 [hep-ph/9909386] [INSPIRE].

[128] C.S. Kim, K.Y. Lee and J.-H. Song, Enhancement of the Higgs pair production at CERN LHC: The MSSM and extra dimension effects, Phys. Rev. D 64 (2001) 015009 [hep-ph/0009231] [INSPIRE].

[129] D.T. Nhung, M. Muhlleitner, J. Streicher and K. Walz, Higher order corrections to the trilinear Higgs self-couplings in the real NMSSM, JHEP 11 (2013) 181 [arXiv:1306.3926] [INSPIRE].

[130] U. Ellwanger, Higgs pair production in the NMSSM at the LHC, JHEP 08 (2013) 077 [arXiv: 1306.5541] [INSPIRE].

[131] C. Han, X. Ji, L. Wu, P. Wu and J.M. Yang, Higgs pair production with SUSY QCD correction: revisited under current experimental constraints, JHEP 04 (2014) 003 [arXiv: 1307.3790] [INSPIRE]. 
[132] J. Cao, Z. Heng, L. Shang, P. Wan and J.M. Yang, Pair production of a $125 \mathrm{GeV}$ Higgs boson in MSSM and NMSSM at the LHC, JHEP 04 (2013) 134 [arXiv:1301.6437] [INSPIRE].

[133] https://twiki.cern.ch/twiki/bin/view/LHCPhysics/Cross Sections.

[134] J. Cao, Y. He, P. Wu, M. Zhang and J. Zhu, Higgs phenomenology in the minimal dilaton model after Run I of the LHC, JHEP 01 (2014) 150 [arXiv:1311.6661] [INSPIRE].

[135] J. Cao, K.-i. Hikasa, W. Wang, J.M. Yang and L.-X. Yu, SUSY dark matter in light of CDMS II results: a comparative study for different models, JHEP 07 (2010) 044 [arXiv: 1005.0761] [INSPIRE].

[136] LUX collaboration, D.S. Akerib et al., First results from the LUX dark matter experiment at the Sanford Underground Research Facility, Phys. Rev. Lett. 112 (2014) 091303 [arXiv: 1310.8214$]$ [INSPIRE].

[137] G.G. Ross and K. Schmidt-Hoberg, The fine-tuning of the generalised NMSSM, Nucl. Phys. B 862 (2012) 710 [arXiv:1108.1284] [INSPIRE].

[138] X. Lu, H. Murayama, J.T. Ruderman and K. Tobioka, A natural Higgs mass in supersymmetry from non-decoupling effects, Phys. Rev. Lett. 112 (2014) 191803 [arXiv: 1308.0792] [INSPIRE].

[139] G.G. Ross, K. Schmidt-Hoberg and F. Staub, The generalised NMSSM at one loop: fine tuning and phenomenology, JHEP 08 (2012) 074 [arXiv: 1205.1509] [INSPIRE].

[140] A. Kaminska, G.G. Ross and K. Schmidt-Hoberg, Non-universal gaugino masses and fine tuning implications for SUSY searches in the MSSM and the GNMSSM, JHEP 11 (2013) 209 [arXiv: 1308.4168] [INSPIRE].

[141] A. Kaminska, G.G. Ross, K. Schmidt-Hoberg and F. Staub, A precision study of the fine tuning in the DiracNMSSM, JHEP 06 (2014) 153 [arXiv:1401.1816] [INSPIRE]. 